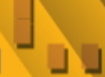

$$
\begin{aligned}
& \text { AVgebra \& } \\
& \text { Number } \\
& \text { Theory }
\end{aligned}
$$

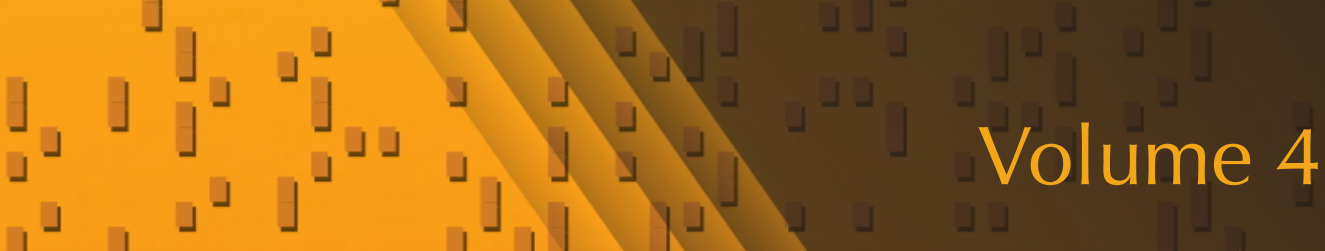

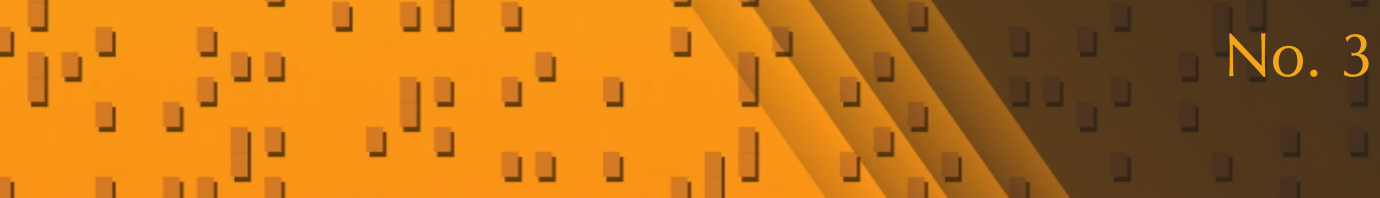

$$
\begin{aligned}
& \text { 」ل }
\end{aligned}
$$

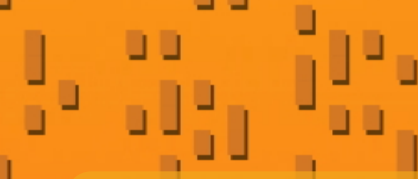

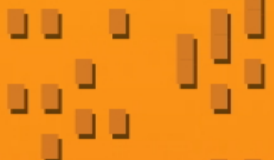

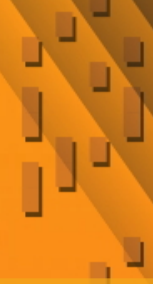

Raccord sur les espaces de Berkovich Jérôme Poineau

\lrcorner

\rfloor

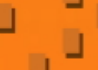

」 


\title{
Raccord sur les espaces de Berkovich
}

\author{
Jérôme Poineau
}

Nous présentons ici quelques résultats autour du problème inverse de Galois. Nous commençons par rappeler la stratégie géométrique classique permettant de démontrer que tout groupe fini est groupe de Galois sur $\mathbf{C}(T)$. Nous l'appliquons dans une autre situation afin de démontrer que, si $M(B)$ désigne le corps des fonctions méromorphes sur une partie $B$, d'un certain type, d'un espace de Berkovich sur un corps, alors l'énoncé précédent reste valable lorsque l'on remplace $\mathbf{C}$ par $M(B)$. On retrouve, en particulier, le fait que tout groupe fini est groupe de Galois sur $K(T)$, lorsque $K$ est un corps valué complet dont la valuation n'est pas triviale.

Dans un second temps, en utilisant une méthode similaire, nous proposons une nouvelle preuve, purement géométrique, dans le langage des espaces de Berkovich sur un anneau d'entiers de corps de nombres, d'un résultat de D. Harbater assurant que tout groupe fini est groupe de Galois sur un corps de séries arithmétiques convergentes, ainsi que quelques généralisations.

Patching on Berkovich spaces. We present a few results related to the inverse Galois problem. First we recall the geometric patching strategy that is used to handle the problem in the complex case. We use it in a different situation to prove that if $M(B)$ is the field of meromorphic functions over a part $B$, satisfying some conditions, of a Berkovich space over a valued field, then every finite group is a Galois group over $M(B)(T)$. From this we derive a new proof of the fact that any finite group is a Galois group over $K(T)$, where $K$ is a complete valued field with nontrivial valuation.

In a second part, we deal with the following theorem by D. Harbater: every finite group is a Galois group over a field of convergent arithmetic power series. We switch to Berkovich spaces over the ring of integers of a number field and use a similar strategy to give a new and purely geometric proof of this theorem, as well as some generalizations.

MSC2000: primary 12F12; secondary 14G22, 14G20, 14G25.

Mots-clefs: problème inverse de Galois, espaces de Berkovich, géométrie analytique $p$-adique, géométrie analytique globale, séries arithmétiques convergentes, inverse Galois problem,

Berkovich spaces, p-adic analytic geometry, global analytic geometry, convergent arithmetic power series.

L'auteur est membre du projet jeunes chercheurs «Espaces de Berkovich» de l'agence nationale de la recherche. 
Introduction 298

1. Stratégie de raccord 299

2. Problème inverse de Galois sur une droite relative 302

3. Problème inverse de Galois sur un disque relatif 313

Annexe A. Théorèmes GAGA relatifs sur un affinoïde 321

Annexe B. La droite de Berkovich sur un anneau d'entiers de corps de nombres 327

Remerciements $\quad 332$

Bibliographie $\quad 333$

\section{Introduction}

Le problème inverse de Galois consiste à montrer que tout groupe fini peut être réalisé comme groupe de Galois sur le corps des nombres rationnels $\mathbf{Q}$. La simplicité de l'énoncé n'augure en rien de la difficulté de la question et sa réponse nous échappe encore à ce jour.

Une stratégie due à D. Hilbert consiste à chercher à réaliser, tout d'abord, un groupe fini $G$ donné comme groupe de Galois sur le corps $\mathbf{Q}(T)$. Ce second problème se prête à une approche géométrique. En effet, supposons que l'on sache construire un revêtement galoisien (ramifié) $X$ de la droite projective $\mathbf{P}_{\mathbf{Q}}^{1}$ de groupe de Galois $G$. L'extension

$$
\mathcal{M}\left(\mathbf{P}_{\mathbf{Q}}^{1}\right)=\mathbf{Q}(T) \rightarrow \mathcal{M}(X)
$$

induite entre les corps de fonctions fournirait alors une solution. Le théorème d'irréductibilité de Hilbert permet ensuite de revenir au problème initial : il assure qu'il est toujours possible de spécialiser une telle extension de façon à obtenir une extension du corps $\mathbf{Q}$ dont le groupe de Galois est encore $G$.

Dans ce texte, nous nous intéressons à des variantes du second problème. Nous commençons par rappeler, dans la section 1, une stratégie classique pour obtenir des revêtements galoisiens : construire localement des revêtements analytiques cycliques, puis raccorder ces revêtements, et enfin montrer que le revêtement obtenu est algébrique.

Dans la deuxième section, nous nous plaçons dans le cadre des espaces analytiques au sens de Berkovich et appliquons la stratégie indiquée. Nous parvenons à démontrer que, lorsque $K$ est un corps ultramétrique complet dont la valuation n'est pas triviale, tout groupe fini est groupe de Galois sur le corps $K(T)$. En particulier, pour tout nombre premier $p$, tout groupe fini est groupe de Galois sur le corps $\mathbf{Q}_{p}(T)$, un corps qui contient $\mathbf{Q}(T)$. La démonstration originale de ce résultat est due à D. Harbater [1987, Corollary 2.4]; elle est écrite dans le cadre de 
la géométrie formelle. Il existe également une preuve dans le cadre de la géométrie rigide, rédigée par Q. Liu [1995] en suivant une idée de J.-P. Serre. Signalons que l'absence, en général, de racines primitives de l'unité de tout ordre complique la première étape. Aussi les deux démonstrations citées font-elles appel aux constructions décrites par D. Saltman [1982]. Dans la preuve que nous proposons ici, en revanche, nous en faisons l'économie : un choix judicieux des lieux où nous construisons les revêtements cycliques nous permet d'avoir recours uniquement à des extensions de Kummer lorsque le corps $K$ est de caractéristique nulle, ou de Kummer et d'Artin-Schreier-Witt lorsqu'il est de caractéristique strictement positive. En toute logique, la simplification de l'arithmétique du problème a un coût et nous utilisons un résultat de géométrie plus compliqué, mais fort naturel : un théorème de type GAGA relatif au-dessus d'un espace affinoïde ( $c f$. annexe A).

La dernière section du texte est consacrée à la construction d'extensions galoisiennes d'un sur-corps de $\mathbf{Q}(T)$ d'un type différent : le corps des fractions de l'anneau $\mathbf{Z}_{1^{-}} \llbracket T \rrbracket$ formé des séries en une variable à coefficients entiers qui convergent sur le disque unité ouvert complexe. Nous en déduisons une nouvelle preuve du résultat de D. Harbater [1988, Corollary 3.8] qui assure que tout groupe fini est groupe de Galois sur ce corps, et l'étendons à tout corps de nombres. La démonstration originale de ce résultat, aboutissement de la série d'articles [Harbater 1984a; 1984b; 1984c; 1988], est ardue et technique; elle est basée sur des manipulations algébriques des anneaux de séries du même type que $\mathbf{Z}_{1^{-}} \llbracket T \rrbracket$. Celle que nous proposons est, en revanche, purement géométrique. La seule difficulté réside dans le fait que le cadre adapté à ce problème est celui, fort naturel mais sans doute encore un peu exotique, de la droite de Berkovich sur un anneau d'entiers de corps de nombres (la construction et les propriétés de cet espace font l'objet de l'annexe B).

Signalons, pour finir, que, contrairement à l'habitude, nous construisons un revêtement d'un ouvert d'un espace affine; les résultats de type GAGA y tombent donc en défaut et nous utiliserons, pour pallier ce manque, le fait que l'ouvert en question soit un espace de Stein.

Notations. Nous désignerons par $\mathbf{N}$ l'ensemble des nombres entiers positifs et par $\mathbf{N}^{*}$ le sous-ensemble formé de ceux qui ne sont pas nuls.

\section{Stratégie de raccord}

Nous rappelons ici une démonstration classique du fait que tout groupe fini est groupe de Galois d'un revêtement de la variété algébrique $\mathbf{P}_{\mathbf{C}}^{1}$. Ce n'est qu'un prétexte pour présenter la stratégie de raccord que nous utiliserons constamment par la suite. 
Considérons tout d'abord le cas des groupes cycliques. Pour disposer de plus de souplesse, nous allons commencer par construire des revêtements de la variété analytique $\mathbf{P}^{1}(\mathbf{C})$, et même des revêtements de petits ouverts de cette variété. Soit $m \in \mathbf{N}^{*}$. Choisissons un point $P$ de $\mathbf{P}^{1}(\mathbf{C})$, une coordonnée locale $z$ au voisinage de ce point, un disque ouvert $D_{P}$ sur lequel elle est définie et un disque fermé $E_{P}$ de rayon strictement positif contenu dans $D_{P}$. Soient $a$ et $b$ deux points distincts de $E_{P}$. Considérons le revêtement connexe et lisse $X_{P}$ du disque $D_{P}$ donné par l'équation

$$
u^{m}=(z-a)(z-b)^{m-1} .
$$

C'est un revêtement galoisien de groupe $\mathbf{Z} / m \mathbf{Z}$. En outre, il est trivial au-dessus du complémentaire du disque $E_{P}$. Remarquons que pour déterminer le groupe de Galois nous avons utilisé le fait que le corps $\mathbf{C}$ contienne une racine primitive $m^{\text {ème }}$ de l'unité.

Nous allons maintenant recoller des revêtements du type précédent afin d'en construire qui possèdent des groupes de Galois finis arbitraires. Fixons un groupe fini $G$. Notons $n$ son ordre et choisissons-en des générateurs $g_{1}, \ldots, g_{t}$, avec $t \in \mathbf{N}^{*}$. Soit $i \in \llbracket 1, t \rrbracket$. Notons $n_{i}$ l'ordre de l'élément $g_{i}$ dans le groupe $G$ et posons $d_{i}=n / n_{i}$. Choisissons un point $P_{i}$ de $\mathbf{P}^{1}(\mathbf{C})$ et construisons, par la méthode du paragraphe précédent, un $\mathbf{Z} / n_{i} \mathbf{Z}$-revêtement $X_{P_{i}}$ au-dessus d'un disque ouvert $D_{P_{i}}$ et trivial hors d'un disque fermé $E_{P_{i}} \subset D_{P_{i}}$. Indexons les feuillets de ce revêtement par les entiers compris entre 0 et $n_{i}-1$ de façon compatible avec l'action du groupe $\mathbf{Z} / n_{i} \mathbf{Z} \simeq\left\langle g_{i}\right\rangle$. Considérons maintenant $\operatorname{Ind}_{\left\langle g_{i}\right\rangle}^{G}\left(X_{P_{i}}\right)$, le $G$-revêtement induit par le $\left\langle g_{i}\right\rangle$-revêtement $X_{P_{i}}$. Rappelons qu'il est constitué topologiquement de $d_{i}$ copies de $X_{P_{i}}$. Nous pouvons envoyer, de façon bijective, les feuillets de ce revêtement sur les éléments du groupe. Pour ce faire, choisissons, dans $G$, des représentants $a_{i, 0}, \ldots, a_{i, d_{i}-1}$ des éléments du quotient $G /\left\langle g_{i}\right\rangle$. L'application qui envoie le feuillet indexé par $k$ de la copie indexée par $l$ de $X_{P_{i}}$ sur l'élément $a_{i, l} g_{i}^{k}$ de $G$ est bijective. Nous pouvons alors décrire l'action du groupe $G$ sur le revêtement $\operatorname{Ind}_{\left\langle g_{i}\right\rangle}^{G}\left(X_{P_{i}}\right)$ de la façon suivante : l'élément $g$ de $G$ envoie le feuillet associé à l'élément $h$ de $G$ sur le feuillet associé à l'élément $h g$.

Notons $D^{\prime}$ le complémentaire de la réunion des disques $E_{P_{1}}, \ldots, E_{P_{t}}$ dans $\mathbf{P}^{1}(\mathbf{C})$. Considérons le $G$-revêtement $\operatorname{Ind}_{\langle e\rangle}^{G}\left(D^{\prime}\right)$ induit par le revêtement trivial de $D^{\prime}$ et indexons ses feuillets par les éléments de $G$ de façon compatible avec l'action de ce groupe.

Raccordons, maintenant, les revêtements que nous venons de construire. Nous supposerons que les disques $D_{P_{i}}$, avec $i \in \llbracket 1, t \rrbracket$, sont deux à deux disjoints. Nous pouvons facilement nous ramener à ce cas en les réduisant, si besoin est. Pour tout élément $i$ de $\llbracket 1, t \rrbracket$, nous recollons alors, au-dessus de l'intersection $D_{P_{i}} \cap D^{\prime}$, les feuillets associés aux mêmes éléments du groupe $G$ ( $c f$. figure 1). Nous obtenons 




FigURE 1. Raccord de revêtements cycliques.

ainsi un revêtement $Y$ de $\mathbf{P}^{1}(\mathbf{C})$ dont on vérifie facilement qu'il est connexe, lisse et galoisien de groupe $G$.

Ainsi avons-nous obtenu une variété analytique complexe $Y$ vérifiant les propriétés requises. Il nous reste à montrer que c'est, en réalité, une variété algébrique. Ce résultat découle du théorème d'existence de Riemann ou, si l'on veut, des théorèmes GAGA de Serre. Nous avons finalement obtenu le résultat suivant :

Théorème 1.1. Tout groupe fini est groupe de Galois d'une extension du corps $\mathbf{C}(T)$.

Pour résumer, rappelons en quelques mots la stratégie de la preuve :

(1) Construire des revêtements cycliques sur de petits ouverts, triviaux au voisinage du bord.

(2) Raccorder ces revêtements.

(3) Montrer que le revêtement obtenu est algébrique.

Harbater l'a développée dans plusieurs contextes et utilisée pour démontrer de nombreux résultats. Nous renvoyons le lecteur désireux d'en savoir plus au texte [Harbater 2003].

La simplicité de cette stratégie de raccord («patching » chez Harbater) invite à l'appliquer dans de nombreux contextes géométriques, pour peu que l'on dispose d'une bonne notion de «petits ouverts » et de théorèmes d'algébricité. Ce n'est pas le cas de la géométrie algébrique, où deux ouverts non vides de la droite projective se coupent toujours, mais ce devrait l'être pour toute géométrie analytique. Dans 
la suite de ce texte, nous illustrerons cette idée en appliquant la stratégie indiquée dans le cadre des espaces de Berkovich sur un corps ultramétrique complet, à la section 2, puis sur un anneau d'entiers de corps de nombres, à la section 3 .

\section{Problème inverse de Galois sur une droite relative}

Soient $k$ un corps muni d'une valeur absolue ultramétrique pour laquelle il est complet et $X$ un espace $k$-analytique géométriquement irréductible. Nous noterons 0 le faisceau structural sur cet espace. Soit $B$ une partie de l'espace $X$.

Notons $Y$ le produit fibré $X \times_{k} \mathbf{P}_{k}^{1, \text { an }}, \pi: Y \rightarrow X$ et $\lambda: Y \rightarrow \mathbf{P}_{k}^{1, \text { an }}$ les morphismes naturels de projection. Signalons que, d'après [Ducros 2009, théorèmes 7.16 et 8.4], l'espace $Y$ est géométriquement irréductible. Notons $Y(B)$ l'image réciproque de $B$ par le morphisme $\pi$.

Dans les numéros 2A, 2B et 2C, nous supposerons que la partie $B$ est compacte (pour les applications, dans la preuve des corollaires 2.13 et 2.14, elle sera même réduite à un point). Nous la munirons alors du faisceau des fonctions surconvergentes, c'est-à-dire du faisceau $j^{-1} \mathfrak{O}$, où $j: B \hookrightarrow X$ désigne l'inclusion. De façon générale, nous utiliserons, sans plus le préciser, le faisceau des fonctions surconvergentes pour toute partie compacte. En particulier, l'espace localement annelé associé à l'espace $Y(B)$ que nous obtiendrons ainsi ne sera autre que le germe $\left(Y, \pi^{-1}(B)\right)$ au sens de Berkovich [1993, §3.4; 1996, §2].

2A. Construction locale de revêtements cycliques. Dans le cas complexe, la construction locale était particulièrement simple, car nous disposions de racines primitives de l'unité de tout ordre. Lorsque nous cherchons à construire un revêtement cyclique dont l'ordre $n$ est premier à l'exposant caractéristique du corps de base, la situation n'est guère plus compliquée, car nous disposons encore de racines primitives $n^{\text {èmes }}$ de l'unité sur certains ouverts. Nous utiliserons alors une extension de Kummer bien choisie. Dans les autres cas, nous ferons appel à la théorie d'ArtinSchreier-Witt. Nous noterons $p$ l'exposant caractéristique du corps $k$.

Fixons une extension finie et séparable $K$ de $k$. Soit $P \in k[T]$ le polynôme minimal unitaire d'un élément primitif de cette extension. Notons $t$ le point de $\mathbf{P}_{k}^{1, \text { an }}$ défini par l'annulation de ce polynôme.

2A1. Extensions de Kummer. Soit $n$ un entier supérieur ou égal à 2 et premier à $p$. Supposons que le corps $K$ contient une racine primitive $n^{\text {ème }}$ de l'unité.

Puisque l'anneau local au point $t$ est hensélien, il contient une racine primitive $n^{\text {ème }}$ de l'unité, que nous noterons $\zeta$. Cette racine est définie sur un voisinage du point $t$, que nous pouvons supposer de la forme

$$
V_{u}=\left\{y \in \mathbf{P}_{k}^{1, \text { an }}|| P(y) \mid \leq u\right\},
$$

avec $u>0$. 
Posons $V=\lambda^{-1}\left(V_{u}\right)$. Nous noterons encore $P$ et $\zeta$ les rétrotirettes des éléments $P$ et $\zeta$ par le morphisme $\lambda$. Remarquons que l'élément $\zeta$ de $O(V)$ est encore une racine primitive $n^{\text {ème }}$ de l'unité.

Soient $U$ un voisinage de $B$ dans $X$ et $\alpha$ un élément de $\mathcal{O}(U)$ nul en tout point de $B$. Posons

$$
Q(S)=S^{n}-P^{n}-\alpha \in \mathcal{O}\left(\pi^{-1}(U)\right)[S] .
$$

Définissons un préfaisceau $\mathscr{F}$ sur $\pi^{-1}(U)$ en posant, pour toute partie ouverte $W$ de $\pi^{-1}(U)$,

$$
\mathscr{F}(W)=\mathcal{O}(W)[S] /(Q(S)),
$$

et en utilisant les morphismes de restriction induits par ceux du faisceau 0 . Le caractère unitaire du polynôme $Q$ assure que $\mathscr{F}$ est un faisceau de $O_{\pi^{-1}(U)}$-algèbres cohérent.

Remarque 2.1. Le faisceau $\mathscr{F}$ est l'image directe du faisceau structural d'une courbe analytique sur $Y$. Celle-ci nous est donnée comme un revêtement ramifié de degré $n$ de $\pi^{-1}(U)$.

Soit $v \in] 0, u]$. Posons

$$
V(B)=\{y \in Y(B)|| P(y) \mid \leq v\} \quad \text { et } \quad V^{\prime}(B)=\{y \in V(B) \mid P(y) \neq 0\} .
$$

Remarquons que le complémentaire de $V^{\prime}(B)$ dans $V(B)$ est fermé dans $Y(B)$.

Proposition 2.2. Il existe un isomorphisme de $\mathrm{O}_{V^{\prime}(B)}$-algèbres

$$
\varphi: \mathscr{F} \rightarrow \mathbb{O}^{n}
$$

tel que, pour tout ouvert $W$ de $V^{\prime}(B)$ et tout élément s de $\mathscr{F}(W)$, nous ayons

$$
\varphi(\zeta s)=\tau(\varphi(s))
$$

où $\tau$ désigne l'automorphisme du faisceau $0^{n}$ qui consiste à faire agir la permutation cyclique $\left(\begin{array}{llll}1 & 2 & \ldots\end{array}\right)$ sur les coordonnées.

Démonstration. Le rayon de convergence de la série

$$
(1+T)^{1 / n}=\sum_{i=0}^{+\infty} C_{1 / n}^{i} T^{i} \in k \llbracket T \rrbracket
$$

est strictement positif. La série

$$
P \sum_{i=0}^{+\infty} C_{1 / n}^{i}\left(\frac{\alpha}{P^{n}}\right)^{i}
$$


définit donc un élément $\omega$ de $\mathscr{O}\left(V^{\prime}(B)\right)$, qui est une racine du polynôme $Q$. Nous avons alors l'égalité

$$
Q(S)=S^{n}-P^{n}-\alpha=\prod_{j=0}^{n}\left(S-\zeta^{j} \omega\right) \quad \text { dans } \mathcal{O}\left(V^{\prime}(B)\right) .
$$

Par conséquent, le morphisme

$$
\mathscr{F} \rightarrow \mathcal{O}^{n}, \quad R(S) \mapsto\left(R(\omega), R\left(\zeta^{-1} \omega\right), \ldots, R\left(\zeta^{-(n-1)} \omega\right)\right)
$$

est un isomorphisme. On vérifie immédiatement qu'il satisfait la condition requise.

Remarque 2.3. La première partie du résultat signifie que le revêtement associé au faisceau $\mathscr{F}$ est trivial au-dessus de $V^{\prime}(B)$. La seconde assure que le groupe $\langle\zeta\rangle \simeq \mathbf{Z} / n \mathbf{Z}$ agit sur le revêtement par une permutation cyclique des feuillets $\mathrm{du}$ lieu trivial.

Nous allons maintenant imposer des conditions sur les données $B$ et $\alpha$ de façon que le faisceau $\mathscr{F}_{F}$ soit associé à un revêtement irréductible.

Définition 2.4. Nous dirons que la partie $B$ de $X$ satisfait la condition CGI si elle est compacte et possède un système fondamental de voisinages affinoïdes géométriquement intègres.

Sous les conditions de cette définition, l'anneau $O(B)$ est intègre et la partie $B$ connexe. En particulier, le principe du prolongement analytique vaut sur $B$ et les anneaux locaux en tous les points de $B$ sont intègres.

Lemme 2.5. Supposons que la partie B de X satisfait la condition CGI. Alors, la partie $V(B)$ de $Y$ possède un système fondamental de voisinages affinö̈des irréductibles.

En particulier, pour tout point $z$ de $V(B)$, le morphisme naturel

$$
\mathrm{O}(V(B)) \rightarrow \mathrm{O}_{z}
$$

est injectif.

Démonstration. Soient $U^{\prime}$ un voisinage affinoïde géométriquement irréductible de $B$ dans $U$ et $v^{\prime}$ un nombre réel strictement supérieur à $v$. La partie de $Y$ définie par

$$
\left\{z \in \pi^{-1}\left(U^{\prime}\right)|| P(z) \mid \leq v^{\prime}\right\}
$$

est alors irréductible, d'après [Ducros 2009, théorème 8.4]. En effet, ce n'est autre que le produit, au-dessus de $k$, de l'espace géométriquement irréductible $U^{\prime}$ par l'espace

$$
\left\{x \in \mathbf{P}_{k}^{1, \text { an }}|| P(x) \mid \leq v^{\prime}\right\},
$$

qui est irréductible, car le polynôme $P$ est irréductible sur $k$. 
La condition CGI assure que l'ensemble des parties de la forme précédente est un système fondamental de voisinages de $V(B)$ dans $Y$.

Nous supposerons désormais que la partie compacte B de X satisfait la condition CGI.

Définition 2.6. Nous dirons que l'élément $\alpha$ de $\mathscr{O}(B)$ satisfait la condition $\mathrm{I}_{n, K}$ s'il existe un point $x$ de $B$ qui vérifie les deux conditions suivantes :

(i) les corps $K$ et $\operatorname{Frac}\left(\mathscr{O}_{x}\right)$ sont linéairement disjoints sur $k$;

(ii) le polynôme $S^{n}-\alpha$ est irréductible sur le corps $\operatorname{Frac}\left(\mathscr{O}_{x}\right) \otimes_{k} K$.

Lemme 2.7. Supposons que l'élément $\alpha$ de $\mathcal{O}(B)$ satisfait la condition $\mathrm{I}_{n, K}$. Alors, le polynôme $Q(S)=S^{n}-P^{n}-\alpha$ est irréductible sur le corps $\operatorname{Frac}(\mathcal{O}(V(B)))$. En particulier, l'anneau $\mathscr{F}(V(B))$ est intègre.

Démonstration. Soit $x$ un point de $B$ satisfaisant les propriétés énoncées dans la définition de la condition $\mathrm{I}_{n, K}$. Puisque les corps $K$ et Frac $\left(O_{x}\right)$ sont linéairement disjoints sur $k$, le polynôme $P$ est irréductible sur $O_{x}$. Il l'est donc encore dans $\mathscr{H}(x)[T]$, puisque le corps $\kappa(x)$ et l'anneau local $O_{x}$ sont henséliens.

Notons $Z(B)$ l'ensemble des points de $Y(B)$ en lesquels la fonction $P$ est nulle. D'après le raisonnement précédent, la trace de la fibre $\pi^{-1}(x)$ sur $Z$ comporte un seul point, que nous noterons $z$. Notons $\mathrm{O}_{Z}$ le faisceau structural sur $Z$. Nous avons alors un isomorphisme

$$
\mathrm{O}_{x}[T] /(P(T)) \stackrel{\sim}{\rightarrow} \mathrm{O}_{Z, z} .
$$

Le polynôme $S^{n}-\alpha$ est donc irréductible sur le corps $\operatorname{Frac}\left(\mathrm{O}_{Z, z}\right)$, isomorphe à $\operatorname{Frac}\left(O_{x}\right) \otimes_{k} K$. Par conséquent, le polynôme $Q(S)$ est irréductible sur le corps $\operatorname{Frac}\left(\mathrm{O}_{z}\right)$.

D'après le lemme 2.5, le morphisme naturel $\mathcal{O}(V(B)) \rightarrow \mathrm{O}_{z}$ est injectif. Par conséquent, le corps Frac $(\mathcal{O}(V(B)))$ est un sous-corps de $\operatorname{Frac}\left(\mathcal{O}_{z}\right)$. On en déduit que le polynôme $Q(S)$ est irréductible sur le corps Frac $(\mathcal{O}(V(B)))$.

Le polynôme $Q(S)$ étant unitaire, l'unicité de la division euclidienne assure que le morphisme

$$
\mathcal{O}(V(B))[S] /(Q(S)) \rightarrow \operatorname{Frac}(\mathcal{O}(V(B)))[S] /(Q(S))
$$

est injectif. Puisque l'anneau au but est intègre, celui à la source, qui n'est autre que l'anneau $\mathscr{F}(V(B))$, l'est également.

Remarque 2.8. Ce résultat signifie que la courbe associée au faisceau $\mathscr{F}$ est intègre, c'est-à-dire réduite et irréductible. 
2A2. Extensions d'Artin-Schreier-Witt. Il nous reste à traiter le cas des groupes cycliques dont l'ordre n'est pas premier à l'exposant caractéristique $p$ du corps $k$. Nous supposons désormais que $p$ est un nombre premier et chercherons à construire un revêtement cyclique d'ordre $n=p^{r}$, où $r$ est un entier supérieur à 1 . Il s'agit essentiellement de remplacer, dans le numéro précédent, la théorie de Kummer par celle d'Artin-Schreier-Witt. Nous nous contenterons d'indiquer les grandes lignes de la preuve.

Dans ce numéro, comme dans le précédent, nous supposerons que la partie compacte $B$ de $X$ satisfait la condition CGI.

Soit $\alpha$ un élément de $O(B)$ nul en tout point de $B$.

Définition 2.9. Nous dirons que l'élément $\alpha$ de $\mathcal{O}(B)$ satisfait la condition $\mathrm{I}_{p, K}$ s'il existe un point $x$ de $B$ qui vérifie les deux conditions suivantes :

(i) les corps $K$ et $\operatorname{Frac}\left(\mathscr{O}_{x}\right)$ sont linéairement disjoints sur $k$;

(ii) le polynôme $S^{p}-\alpha$ est irréductible sur le corps $\operatorname{Frac}\left(O_{x}\right) \otimes_{k} K$.

Soit $v>0$. Posons

$$
V(B)=\{y \in Y(B)|| P(y) \mid \leq v\} \quad \text { et } \quad V^{\prime}(B)=\{y \in V(B) \mid P(y) \neq 0\} .
$$

Soit $W_{r}$ l'anneau des vecteurs de Witt de longueur $r$ sur $\mathcal{O}(V(B))\left[S_{0}, \ldots, S_{r-1}\right]$. Posons

$$
S=\left(S_{0}, \ldots, S_{r-1}\right) \in W_{r}
$$

et, pour tout élément $a$ de $\mathcal{O}(V(B))\left[S_{0}, \ldots, S_{r-1}\right]$,

$$
\{a\}=(a, 0, \ldots, 0) \in W_{r} .
$$

Pour tout $i \in \llbracket 0, r-1 \rrbracket$, définissons un polynôme $Q_{i}\left(S_{0}, \ldots, S_{r-1}\right)$ à coefficients dans $O(V(B))$ par la formule

$$
\left(Q_{0}, \ldots, Q_{r-1}\right)=F(S)-\{P\}^{p-1} S-\{\alpha\} \text { dans } W_{r} .
$$

Le groupe $\mathbf{Z} / p^{r} \mathbf{Z}$ agit sur l'anneau $\mathcal{O}(V(B))\left[S_{0}, \ldots, S_{r-1}\right] /\left(Q_{0}, \ldots, Q_{r-1}\right)$ en laissant stable $\mathcal{O}(V(B))$ et en envoyant $S_{i}$, pour $i \in \llbracket 0, r-1 \rrbracket$, sur la $(i+1)^{\text {ème }}$ coordonnée du vecteur $S+\{P\}$ dans $W_{r}$. Par analogie avec la construction précédente, nous définissons un faisceau sur $V(B)$ par

$$
\mathscr{F}=\mathcal{O}\left[S_{0}, \ldots, S_{r-1}\right] /\left(Q_{0}, \ldots, Q_{r-1}\right) .
$$

Les propriétés des vecteurs de Witt montrent que, pour tout $i \in \llbracket 0, r-1 \rrbracket$, nous avons

$$
Q_{i}=S_{i}^{p}-P^{(p-1) p^{i}} S_{i} \bmod \left(\alpha, Q_{0}, \ldots, Q_{i-1}\right) .
$$

Soit $y \in V^{\prime}(B)$. L'image $S_{0}^{p}-P^{p-1} S_{0}$ du polynôme $Q_{0}\left(S_{0}\right)$ dans le corps résiduel $\kappa(y)$ de l'anneau local $O_{y}$ est scindé à racines simples. Puisque cet anneau 
local est hensélien, le polynôme $P_{0}\left(S_{0}\right)$ possède $p$ racines simples dans $O_{y}$. En raisonnant par récurrence sur le nombre de variables, on montre ainsi que le système d'équations polynomiales donné par $Q_{0}, \ldots, Q_{r-1}$ possède exactement $p^{r}$ racines $\omega_{1}, \ldots, \omega_{p^{r}}$ dans $\mathbb{O}_{y}^{r}$ et que le morphisme

$$
\psi: \mathscr{O}_{y}\left[S_{0}, \ldots, S_{r-1}\right] /\left(Q_{0}, \ldots, Q_{r-1}\right) \rightarrow \mathscr{O}_{y}^{p^{r}}, \quad R\left(S_{0}, \ldots, S_{r-1}\right) \mapsto\left(R\left(\omega_{i}\right)\right)_{1 \leq i \leq p^{r}}
$$

est un isomorphisme. On en déduit que le revêtement est trivial sur $V^{\prime}(B)$, comme à la proposition 2.2.

Supposons que l'élément $\alpha$ de $\mathscr{O}(B)$ satisfait la condition $\mathrm{I}_{p, K}$. L'énoncé du lemme 2.7 vaut alors encore. Pour le démontrer, l'on remplace simplement les arguments de la théorie de Kummer par ceux de la théorie d'Artin-Schreier-Witt. L'argument-clé consiste à utiliser le fait que le polynôme $S^{p}-\alpha$ est irréductible sur $O_{x}$, où $x$ est un point de $B$ satisfaisant les propriétés énoncées dans la définition de la condition $\mathrm{I}_{p, K}$, et à en déduire que le polynôme $S^{p}-P^{p-1} S-\alpha$ est irréductible sur $O_{z}$, où $z$ désigne l'unique point de la fibre $\pi^{-1}(x)$ en lequel $P$ s'annule.

2B. Raccord et retour à l'algèbre. Soit $G$ un groupe fini. Soient $g_{1}, \ldots, g_{t}$, avec $t \in \mathbf{N}^{*}$, des générateurs du groupe $G$. Nous pouvons les choisir de façon que, pour tout élément $i$ de $\llbracket 1, t \rrbracket$, il existe un nombre premier $p_{i}$ et un entier $r_{i} \geq 1$ tels que le sous-groupe de $G$ engendré par $g_{i}$ soit cyclique d'ordre $p_{i}^{r_{i}}$. Nous supposerons qu'il existe $s \in \llbracket 1, t \rrbracket$ tel que $p_{i} \neq p$ pour tout $i \leq s$ et $p_{i}=p$ pour tout $i \geq s+1$.

Nous nous plaçons sous les hypothèses suivantes :

- la partie $B$ de $X$ satisfait la condition CGI;

- pour tout élément $i$ de $\llbracket 1, s \rrbracket$, il existe une extension séparable $K_{i}$ de $k$ contenant une racine primitive $\left(p_{i}^{r_{i}}\right)^{\text {ème }}$ de l'unité et un élément $\alpha_{i}$ de $\mathcal{O}(B)$ qui satisfait la condition $\mathrm{I}_{p_{i}^{r_{i}}, K_{i}}$;

- pour tout élément $i$ de $\llbracket s+1, t \rrbracket$, il existe une extension séparable $K_{i}$ de $k$ et un élément $\alpha_{i}$ de $\mathcal{O}(B)$ qui satisfait la condition $\mathrm{I}_{p, K_{i}}$;

- les corps $K_{1}, \ldots, K_{t}$ sont deux à deux non isomorphes.

Supposons que les éléments $\alpha_{1}, \ldots, \alpha_{t}$ sont nuls en tout point de $B$. Soit $i$ un élément de $\llbracket 1, t \rrbracket$. Construisons par la méthode du numéro $2 \mathrm{~A} 1$ ou $2 \mathrm{~A} 2$ un revêtement galoisien de groupe $\mathbf{Z} / p_{i}^{r_{i}} \mathbf{Z}$. Il est défini au-dessus d'une partie $V_{i}$ et trivial au-dessus d'une partie $V_{i}^{\prime}$. Notons $\operatorname{Ind}_{\left\langle g_{i}\right\rangle}^{G}\left(V_{i}\right)$ le $G$-revêtement induit.

Puisque les corps $K_{1}, \ldots, K_{t}$ sont deux à deux non isomorphes, nous pouvons choisir les parties $V_{1}, \ldots, V_{t}$ de façon qu'elles soient deux à deux disjointes (il suffit de choisir des éléments $v$ assez petits). Pour $i \in \llbracket 1, t \rrbracket$, posons $V_{i}^{\prime \prime}=V_{i} \backslash V_{i}^{\prime}$. C'est une partie fermée de $Y(B)$. Posons

$$
Y^{\prime}(B)=Y(B) \backslash \bigcup_{1 \leq i \leq t} V_{i}^{\prime \prime}
$$


et considérons le $G$-revêtement $\operatorname{Ind}_{\langle e\rangle}^{G}\left(Y^{\prime}(B)\right)$ induit par le revêtement trivial audessus de $Y^{\prime}(B)$.

Raccordons, à présent, les différents revêtements selon les relations entre les éléments du groupe $G$, par la méthode décrite à la section 1 . Nous obtenons un revêtement de $Y(B)$, galoisien de groupe $G$. On montre à l'aide du lemme 2.7 et de son analogue dans le cas des revêtements d'Artin-Schreier-Witt, qu'il est intègre. Un théorème du type GAGA (corollaire A.6) assure qu'il est algébrique. En passant aux corps de fonctions, nous obtenons donc finalement une extension finie et galoisienne de groupe $G$

$$
M(Y(B))=\operatorname{Frac}(O(B))(T) \rightarrow L,
$$

où $M$ désigne le faisceau des fonctions méromorphes. La construction que nous avons menée étant purement géométrique, on se convainc aisément que cette extension est régulière, c'est-à-dire que le corps $\operatorname{Frac}(\mathcal{O}(B))$ est algébriquement fermé dans le corps $L$.

Théorème 2.10. Il existe une extension finie du corps $\operatorname{Frac}(O(B))(T)$ qui est régulière et galoisienne de groupe de Galois G.

2C. Conclusion. Regroupons les résultats que nous avons obtenus jusqu'ici.

Théorème 2.11. Soient $k$ un corps muni d'une valeur absolue ultramétrique pour laquelle il est complet. Soient $X$ un espace $k$-analytique et $B$ une partie compacte de $X$ qui possède un système fondamental de voisinages affinoïdes géométriquement intègres. Supposons que pour tout nombre premier q différent de la caractéristique du corps $k$ et tout entier $r \in \mathbf{N}^{*}$, il existe une famille infinie $\mathcal{K}_{q^{r}}$ de corps deux à deux non isomorphes satisfaisant les propriétés suivantes:

(i) tout élément de $\mathcal{K}_{q^{r}}$ est une extension finie et séparable de $k$ contenant une racine primitive $\left(q^{r}\right)^{\text {ème }}$ de l'unité;

(ii) pour tout élément $K$ de $\mathscr{K}_{q^{r}}$, il existe un élément $x$ de $B$ et un élément $\alpha$ de $\mathcal{O}(B)$ nul en tout point de $B$ tels que les corps $K$ et $\operatorname{Frac}\left(O_{x}\right)$ soient linéairement disjoints et le polynôme $S^{q^{r}}-\alpha$ soit irréductible sur leur compositum.

Si la caractéristique du corps $k$ est un nombre premier p, supposons en outre qu'il existe une famille infinie $\mathscr{K}_{p}$ de corps deux à deux non isomorphes satisfaisant les propriétés suivantes:

(i) tout élément de $\mathscr{K}_{p}$ est une extension finie et séparable de $k$;

(ii) pour tout élément $K$ de $\mathscr{K}_{p}$, il existe un élément $x$ de $B$ et un élément $\alpha$ de $\mathcal{O}(B)$ nul en tout point de $B$ tels que les corps $K$ et $\operatorname{Frac}\left(O_{x}\right)$ soient linéairement disjoints et le polynôme $S^{p}-\alpha$ soit irréductible sur leur compositum.

Alors, tout groupe fini est groupe de Galois d'une extension finie et régulière du corps $\operatorname{Frac}(O(B))(T)$. 
Remarque 2.12. Ce théorème ne contient malheureusement aucun résultat qui ne soit déjà connu. En effet, le corps Frac $(\mathcal{O}(B))$ contient toujours un corps complet pour une valeur absolue non triviale (un corps de séries de Laurent engendré par l'un des éléments $\alpha$ lorsque $k$ est trivialement valué) et, sur un tel corps, le résultat est dû à Harbater [1987, Corollary 2.4].

Nous allons, à présent, appliquer ce résultat général dans des cas particuliers.

Corollaire 2.13. Soit $k$ un corps muni d'une valeur absolue ultramétrique non triviale pour laquelle il est complet. Alors, tout groupe fini est groupe de Galois d'une extension finie et régulière du corps $k(T)$.

Démonstration. Appliquons le théorème 2.11 en choisissant pour espace $X$ la droite analytique $\mathbf{A}_{k}^{1, \text { an }}$, dont nous noterons $U$ la variable, et pour partie $B$ le point 0 . Soit $K$ une extension finie du corps $k$. Choisissons pour point $x$ le point $0:$ l'anneau local $\mathscr{O}_{0}$ est l'anneau des séries en une variable à coefficients dans $k$ de rayon de convergence strictement positif. Par conséquent, les corps $K$ et $\operatorname{Frac}\left(\mathscr{O}_{0}\right)$ sont linéairement disjoints sur $k$. Choisissons pour fonction $\alpha$ la fonction $U$ : pour tout entier $n \geq 1$, le polynôme $S^{n}-U$ est irréductible sur le corps $\operatorname{Frac}\left(\mathscr{O}_{0}\right) \otimes_{k} K$, qui est un sous-corps de $K((U))$, par le théorème d'Eisenstein. Les hypothèses du théorème 2.11 sont donc satisfaites.

Soit $G$ un groupe fini. Il existe une extension finie et régulière du corps

$$
\operatorname{Frac}(O(B))(T)=\operatorname{Frac}\left(O_{0}\right)(T),
$$

qui est galoisienne de groupe $G$. Puisque l'anneau local $O_{0}$ est composé de séries convergentes et que le corps $k$ n'est pas trivialement valué, toute variété qui possède un point sur Frac $\left(\mathrm{O}_{0}\right)$ en possède un sur $k$. En utilisant le théorème de BertiniNoether [Fried et Jarden 2008, Proposition 10.4.2], on montre alors que l'on peut spécialiser l'extension précédente en une extension finie et régulière du corps $k(T)$ qui est galoisienne de groupe $G$.

Rappelons qu'un corps $k$ est dit fertile ${ }^{1}$ si tout $k$-schéma de type fini qui possède un point sur $k((U))$ en possède un sur $k$ (l'on peut démontrer que cela équivaut à demander que toute $k$-courbe lisse qui possède un point sur $k$ en possède une infinité). F. Pop a démontré que, si $k$ est un corps fertile, tout groupe fini est groupe de Galois d'une extension finie et régulière du corps $k(T)$ [Pop 1996, Main Theorem A].

Corollaire 2.14. Soit $k$ un corps. Alors, tout groupe fini est groupe de Galois d'une extension finie et régulière du corps $k((U))(T)$. En particulier, si le corps $k$ est fertile ou contient un corps fertile, tout groupe fini est groupe de Galois d'une extension finie et régulière du corps $k(T)$.

1. Nous empruntons ce terme à L. Moret-Bailly [2001]. Les corps fertiles sont connus sous beaucoup d'autres noms. Ils ont été introduits par F. Pop [1996] sous l'appellation de «large fields ». 
Démonstration. Munissons le corps $k$ de la valuation triviale et considérons la même situation que dans la preuve précédente. Nous avons alors $\mathscr{O}(B)=\mathscr{O}_{0}=k \llbracket U \rrbracket$ et le théorème 2.11 fournit le résultat annoncé.

Lorsque le corps $k$ est fertile, le théorème de Bertini-Noether permet de spécialiser l'extension précédente en une extension de $k(T)$ possédant les mêmes propriétés. La régularité de l'extension de $k(T)$ permet d'obtenir, par produit tensoriel, pour tout corps $L$ contenant $k$, une extension de $L(T)$ possédant encore les propriétés requises.

Remarque 2.15. Le résultat du second corollaire contient le résultat du premier, puisque tout corps complet pour une valeur absolue ultramétrique non triviale est fertile. Cependant, la preuve de ce dernier énoncé étant assez difficile (on peut, par exemple, le démontrer en utilisant l'approximation d'Artin), nous avons choisi de proposer une preuve directe du corollaire 2.13.

2D. Cas de la valuation triviale. Lorsque le corps $k$ est trivialement valué, nous pouvons obtenir des résultats plus généraux. Nous indiquons simplement ici les modifications à apporter au raisonnement qui précède.

Supposons que le corps $k$ est muni de la valeur absolue triviale. Pour tout $n \in$ $\mathbf{N}$, nous pouvons alors définir une application, appelée flot [Poineau 2008, 1.3], de $\mathbf{A}_{k}^{n, \text { an }} \times \mathbf{R}_{+}^{*}$ dans $\mathbf{A}_{k}^{n, \text { an }}$ de la façon suivante. Soient $x$ un point de $\mathbf{A}_{k}^{n \text {,an }}-$ il est associé à une semi-norme multiplicative $|.|_{x}$ sur $k\left[T_{1}, \ldots, T_{n}\right]$ qui induit la valeur absolue triviale sur $k$ - et $\varepsilon$ un nombre réel strictement positif. L'image du couple $(x, \varepsilon)$ est le point de $\mathbf{A}_{k}^{n \text {,an }}$ associée à la semi-norme multiplicative $|\cdot|_{x}^{\varepsilon}$. Par restriction à la source et au but, nous pouvons encore définir le flot sur tout fermé de Zariski d'un espace affine analytique. Signalons qu'une fonction définie au voisinage d'un point se prolonge, et ce de façon unique, à un voisinage de sa trajectoire sous le flot (ibid., proposition 1.3.10).

Nous considérerons désormais un espace analytique $X$ qui est un fermé de Zariski d'un espace affine analytique et une partie ouverte $B$ de $X$. Nous définissons comme précédemment $Y, \pi$ et $\lambda$.

Expliquons comment adapter les constructions locales du numéro 2A. Comme alors, choisissons une extension finie et séparable $K$ de $k$. Soit $P \in k[T]$ le polynôme minimal unitaire d'un élément primitif de cette extension et notons $t$ le point de $\mathbf{P}_{k}^{1, \text { an }}$ défini par l'annulation de ce polynôme.

Reprenons, à présent, le raisonnement du numéro $2 \mathrm{~A} 1$. À cet effet, choisissons un entier $n$ supérieur ou égal à 2 et premier à $p$ et supposons que le corps $K$ contient une racine primitive $n^{\text {ème }}$ de l'unité. Par hensélianité, elle se relève, dans l'anneau local au point $t$, en une racine primitive $n^{\text {ème }}$ de l'unité, que nous noterons $\zeta$. Les propriétés du flot assurent qu'elle est définie sur l'ouvert

$$
\left\{y \in \mathbf{P}_{k}^{1, \text { an }}|| P(y) \mid<1\right\} .
$$


Soit $\alpha$ un élément de $\mathcal{O}(B)$. Insistons sur le fait que nous ne supposons plus qu'il soit nul en tout point de $B$. Nous définissons alors un faisceau $\mathscr{F}$, comme précédemment, au-dessus de l'ouvert

$$
V_{t}(B)=\{y \in Y(B)|| P(y) \mid<1\} .
$$

Puisque le corps $k$ est trivialement valué, le rayon de convergence de la série $(1+T)^{1 / n}$ est égal à 1 et le revêtement associé à $\mathscr{F}$ est trivial au-dessus de l'ouvert

$$
V_{t}^{\prime}(B)=\left\{\left.y \in V(B)|| \alpha(y)|<| P(y)\right|^{n}\right\} .
$$

Supposons, en outre, que l'élément $\alpha$ est de valeur absolue strictement inférieure à 1 en tout point de $B$. Alors, le complémentaire de la partie $V_{t}^{\prime}(B)$ dans $V_{t}(B)$ est fermé dans $Y(B)$.

Pour assurer l'irréductibilité du revêtement associé au faisceau $\mathscr{F}$, nous remplaçons la condition CGI par la condition suivante : l'ouvert $B$ est limite inductive d'espaces affinoïdes géométriquement intègres. Le résultat du lemme 2.7 vaut alors encore.

Passons aux résultats du numéro 2A2. Supposons donc que $p$ est un nombre premier et considérons un entier $n$ de la forme $p^{r}$, avec $r \in \mathbf{N}^{*}$. Soit $\alpha$ un élément de $\mathcal{O}(B)$ et posons, de nouveau,

$$
V_{t}(B)=\{y \in Y(B)|| P(y) \mid<1\} .
$$

Les propriétés du flot permettent de préciser le domaine de définition des racines du polynôme $P_{0}\left(S_{0}\right)$. Notons $B_{0}$ le lieu d'annulation de $\alpha$ dans $B$. Supposons qu'il ne soit pas vide et que pour tout point $b$ de $B$ vérifiant $|\alpha(b)|<1$ et tout voisinage $B_{+}$ de $B_{0}$, le flot joigne le point $b$ à un point de $B_{+}$(c'est en particulier le cas dès que la partie $B$ est stable par le flot, que l'élément $\alpha$ est de valeur absolue strictement inférieure à 1 en tout point de $B$ et s'annule sur $B$ ). Posons

$$
V_{t}^{\prime}(B)=\{y \in V(B)|| \alpha(y) \mid<1 \text { et }|P(y)|<1\} .
$$

Les propriétés du flot assurent que le revêtement associé à $\mathscr{F}$ est encore trivial sur une partie $V_{t}^{\prime}(B)$ de $V_{t}(B)$ dont le complémentaire dans $V_{t}(B)$ est fermé. Si l'élément $\alpha$ est de valeur absolue strictement inférieure à 1 en tout point de $B$, nous pouvons même choisir la partie $V_{t}^{\prime}(B)$ de façon que son complémentaire dans $V_{t}(B)$ soit fermé dans $Y(B)$.

Dans la preuve du théorème 2.10, il faut finalement remplacer le résultat de type GAGA du corollaire A.6 par celui du corollaire A.7.

Nous obtenons finalement le résultat suivant :

Théorème 2.16. Soient $k$ un corps. Munissons-le de la valeur absolue triviale. Soient $X$ un espace de Zariski d'un espace affine $k$-analytique et $B$ une partie 
ouverte de X stable par le flot qui soit limite inductive d'espaces affinoïdes géométriquement intègres. Supposons que pour tout nombre premier q différent de la caractéristique du corps $k$ et tout entier $r \in \mathbf{N}^{*}$, il existe une famille infinie $\mathscr{K}_{q^{r}}$ de corps deux à deux non isomorphes satisfaisant les propriétés suivantes:

(i) tout élément de $\mathscr{K}_{q^{r}}$ est une extension finie et séparable de $k$ contenant une racine primitive $\left(q^{r}\right)^{\text {ème }}$ de l'unité ;

(ii) pour tout élément $K$ de $\mathscr{K}_{q^{r}}$, il existe un élément $x$ de $B$ et un élément $\alpha$ de $\mathrm{O}(B)$ de valeur absolue strictement inférieure à 1 en tout point de $B$ et qui s'annule sur $B$ tels que les corps $K$ et $\operatorname{Frac}\left(O_{x}\right)$ soient linéairement disjoints et le polynôme $S^{q^{r}}-\alpha$ soit irréductible sur leur compositum.

Si la caractéristique du corps $k$ est un nombre premier $p$, supposons en outre qu'il existe une famille infinie $\mathscr{K}_{p}$ de corps deux à deux non isomorphes satisfaisant les propriétés suivantes:

(i) tout élément de $\mathscr{K}_{p}$ est une extension finie et séparable de $k$;

(ii) pour tout élément $K$ de $\mathscr{K}_{p}$, il existe un élément $x$ de $B$ et un élément $\alpha$ de $\mathrm{O}(B)$ de valeur absolue strictement inférieure à 1 en tout point de $B$ et qui $s^{\prime}$ 'annule sur $B$ tels que les corps $K$ et $\operatorname{Frac}\left(\mathbb{O}_{x}\right)$ soient linéairement disjoints et le polynôme $S^{p}-\alpha$ soit irréductible sur leur compositum.

Alors, tout groupe fini est groupe de Galois d'une extension finie et régulière du corps $\operatorname{Frac}(O(B))(T)$.

Remarque 2.17. De nouveau, le résultat de ce théorème est connu, puisque le corps Frac $(\mathcal{O}(B))$ contient un corps de séries de Laurent sur $k$ (engendré par l'un des éléments $\alpha$ ).

Corollaire 2.18. Soit $k$ un corps. Notons

$$
k_{+\infty, 1^{-}} \llbracket U_{1}, U_{2} \rrbracket
$$

le sous-anneau de $k\left[U_{1}\right] \llbracket U_{2} \rrbracket$ composé des séries de la forme

$$
\sum_{n \geq 0} a_{n}\left(U_{1}\right) U_{2}^{n}
$$

qui vérifient la condition

$$
\forall r>0, \forall s \in\left[0,1\left[, \lim _{n \rightarrow+\infty}\left(r^{\operatorname{deg}\left(a_{n}\right)} s^{n}\right)=0 .\right.\right.
$$

Alors, tout groupe fini est groupe de Galois d'une extension finie et régulière du corps $\operatorname{Frac}\left(k_{+\infty, 1^{-}} \llbracket U_{1}, U_{2} \rrbracket\right)(T)$. 
Démonstration. Appliquons le théorème 2.11 en choisissant pour espace $X$ l'espace analytique de dimension deux $\mathbf{A}_{k}^{2 \text {,an }}$, dont nous noterons $U_{1}$ et $U_{2}$ les variables, et pour partie $B$ le disque ouvert relatif de rayon 1 au-dessus de $\mathbf{A}_{k}^{1, \text { an }}$ :

$$
B=\left\{x \in \mathbf{A}_{k}^{2, \text { an }}|| U_{2}(x) \mid<1\right\} .
$$

Cette partie est stable par le flot et nous avons $\mathcal{O}(B)=k_{+\infty, 1^{-}} \llbracket U_{1}, U_{2} \rrbracket$.

Soit $K$ une extension finie du corps $k$. Choisissons pour point $x$ le point de coordonnées $(0,0)$ : l'anneau local $O_{x}$ est isomorphe à $k \llbracket U_{1}, U_{2} \rrbracket$. Par conséquent, les corps $K$ et $\operatorname{Frac}\left(\mathscr{O}_{x}\right)$ sont linéairement disjoints sur $k$. Choisissons pour fonction $\alpha$ la fonction $U_{2}$ : pour tout entier $n \geq 1$, le polynôme $S^{n}-U_{2}$ est irréductible sur le $\operatorname{corps} \operatorname{Frac}\left(O_{x}\right) \otimes_{k} K \simeq K \llbracket U_{1}, U_{2} \rrbracket$. Les hypothèses du théorème 2.11 sont donc satisfaites. On en déduit le résultat attendu.

Ce dernier énoncé peut surprendre, puisqu'il découle directement du corollaire 2.14. En effet, le corps $\operatorname{Frac}\left(k_{r^{-}, 1^{-}} \llbracket U_{1}, U_{2} \rrbracket\right)$ contient le corps $k\left(\left(U_{2}\right)\right)$. Il présente cependant un intérêt dans le cadre de l'analogie entre corps de fonctions et corps de nombres. La droite analytique sur un corps trivialement valué est proche, à bien des égards, du spectre analytique — espace analytique de dimension 0 - d'un anneau d'entiers de corps de nombres (voir annexe B). Il semble donc raisonnable d'envisager que le résultat du corollaire précédent reste vrai en remplaçant l'anneau $k_{r^{-}, 1^{-}} \llbracket U_{1}, U_{2} \rrbracket$ par l'anneau du disque ouvert de rayon 1 au-dessus du spectre d'un anneau d'entiers de corps de nombres. Signalons que les constructions effectuées peuvent effectivement être menées dans ce cadre. Pour conclure, seuls manquent encore les théorèmes du type GAGA sur les espaces de Berkovich au-dessus des anneaux d'entiers de corps de nombres.

Conjecture 2.19. Soient $K$ un corps de nombres, A l'anneau de ses entiers et $\Sigma_{\infty}$ l'ensemble des plongements de $K$ dans $\mathbf{C}$. Notons $A_{1^{-}} \llbracket X \rrbracket$ le sous-anneau de $A \llbracket X \rrbracket$ composé des séries $f$ telles que, pour tout $\sigma \in \Sigma_{\infty}$, la série à coefficients complexes $\sigma(f)$ a un rayon de convergence supérieur ou égal à 1 . Alors, tout groupe fini est groupe de Galois d'une extension finie et régulière du corps $\operatorname{Frac}\left(A_{1-} \llbracket X \rrbracket\right)(T)$.

Remarque 2.20. Nous ignorons si le corps $\operatorname{Frac}\left(A_{1^{-}} \llbracket X \rrbracket\right)$ est fertile, même lorsque l'anneau $A$ est l'anneau $\mathbf{Z}$ des entiers.

\section{Problème inverse de Galois sur un disque relatif}

Soit $A$ un anneau d'entiers de corps de nombres. Nous allons maintenant appliquer la stratégie de raccord décrite à la section 1 dans le cadre des espaces de Berkovich sur $A$ (voir annexe B). Plus précisément, un groupe fini $G$ étant donné, 
nous allons construire un revêtement galoisien de groupe $G$ du disque

$$
\mathbf{D}=\left\{x \in \mathbf{A}_{A}^{1, \text { an }}|| T(x) \mid<1\right\} .
$$

Cela indique que la troisième étape de notre démonstration différera fondamentalement de la troisième étape de la démonstration du cas complexe. En effet, le disque $\mathbf{D}$ est un espace affine et non plus projectif comme l'était $\mathbf{P}^{1}(\mathbf{C})$. En particulier, les théorèmes GAGA n'y sont pas valables. Nous utiliserons, pour les remplacer, le caractère Stein du disque $\mathbf{D}$.

Nous noterons $X=\mathbf{A}_{A}^{1, \text { an }}$ et, pour tout idéal maximal $\mathfrak{m}$ de $A$,

$$
\left.\left.\mathbf{D}_{\mathfrak{m}}=\mathbf{D} \cap \pi^{-1}\left(\left[a_{0}, \tilde{a}_{\mathfrak{m}}\right]\right) \quad \text { et } \quad \mathbf{D}_{\mathfrak{m}}^{\prime}=\mathbf{D} \cap \pi^{-1}(] a_{0}, \tilde{a}_{\mathfrak{m}}\right]\right) .
$$

Ces deux parties sont connexes.

3A. Construction locale de revêtements cycliques. Dans le cas complexe, la construction locale était particulièrement simple car nous disposions de racines primitives de l'unité de tout ordre. Elle ne sera guère plus difficile ici puisque, comme nous allons l'expliquer, un entier $n \geq 1$ étant donné, il existe toujours une branche de $M(A)$, et même une infinité, dont l'anneau des fonctions contient une racine primitive $n^{\text {ème }}$ de l'unité.

Soient $n \geq 1$ un entier, $p$ un nombre premier congru à 1 modulo $n$ et $\mathfrak{m}$ un idéal maximal de $A$ contenant $p$. Notons $\hat{A}_{\mathfrak{m}}$ le complété de l'anneau $A$ pour la topologie $\mathfrak{m}$-adique. Soit $\pi_{\mathfrak{m}}$ une uniformisante de l'anneau $\hat{A}_{\mathfrak{m}}$. Posons

$$
Q(S)=S^{n}-\pi_{\mathfrak{m}}^{n}-T \in \mathcal{O}\left(\mathbf{D}_{\mathfrak{m}}^{\prime}\right)[S] .
$$

Définissons un préfaisceau $\mathscr{F}$ sur $D_{\mathfrak{m}}^{\prime}$ en posant, pour toute partie ouverte $W$ de $D_{\mathfrak{m}}^{\prime}$,

$$
\mathscr{F}(W)=\mathcal{O}(W)[S] /(Q(S)),
$$

et en utilisant les morphismes de restriction induits par ceux du faisceau $\mathrm{O}$. Le caractère unitaire du polynôme $Q$ assure que $\mathscr{F}$ est un faisceau de $0_{D_{\mathfrak{m}}^{\prime}}$-algèbres cohérent. Nous considérons ce faisceau comme l'image directe du faisceau d'un revêtement fini de $\mathbf{D}_{\mathfrak{m}}^{\prime}$.

Le résultat classique qui suit explique le choix des entiers $n$ et $p$.

Lemme 3.1. L'anneau $\mathbf{Z}_{p}$ contient une racine primitive $n^{\text {ème }}$ de l'unité et, pour tout $i \in \mathbf{N}$, nous avons $C_{1 / n}^{i} \in \mathbf{Z}_{p}$.

Soit $\zeta \in \hat{A}_{\mathfrak{m}}$ une racine primitive $n^{\text {ème }}$ de l'unité. Posons

$$
U=\left\{\left.x \in \mathbf{D}_{\mathfrak{m}}^{\prime}|| T(x)|<| \pi_{\mathfrak{m}}(x)\right|^{n}\right\} .
$$

Le résultat suivant affirme que le revêtement de $\mathbf{D}_{\mathfrak{m}}^{\prime}$ associé au faiseau $\mathscr{F}$ est trivial au-dessus de l'ouvert $U$. 
Proposition 3.2. Il existe un isomorphisme de $\mathrm{O}_{U}$-algèbres

$$
\varphi: \mathscr{F} \rightarrow \mathbb{O}^{n}
$$

tel que, pour tout ouvert $V$ de $U$ et tout élément s de $\mathscr{F}(V)$, nous ayons

$$
\varphi(\zeta s)=\tau(\varphi(s))
$$

où $\tau$ désigne l'automorphisme du faisceau $\mathbb{O}^{n}$ qui consiste à faire agir la permutation cyclique $\left(\begin{array}{lll}1 & 2 \ldots n\end{array}\right)$ sur les coordonnées.

Démonstration. En utilisant le lemme précédent, on montre que la fonction $\pi_{\mathfrak{m}}^{-n} T$ possède une racine $n^{\text {ème }}$ dans $\mathcal{O}(U)$. Nous la noterons $\omega$. On en déduit l'égalité

$$
Q(S)=S^{n}-\pi_{\mathfrak{m}}^{n}-T=\prod_{j=0}^{n}\left(S-\pi_{\mathfrak{m}} \zeta^{j} \omega\right) \quad \text { dans } \mathcal{O}(U)[S] .
$$

Par conséquent, le morphisme

$$
\mathscr{F} \rightarrow \mathcal{O}^{n}, \quad R(S) \mapsto\left(R\left(\pi_{\mathfrak{m}} \omega\right), R\left(\pi_{\mathfrak{m}} \zeta^{-1} \omega\right), \ldots, R\left(\pi_{\mathfrak{m}} \zeta^{-(n-1)} \omega\right)\right)
$$

est un isomorphisme. On vérifie immédiatement qu'il satisfait la condition requise.

Démontrons, à présent, que le revêtement est irréductible.

Lemme 3.3. Le polynôme $Q(S)=S^{n}-\pi_{\mathfrak{m}}^{n}-T$ est irréductible sur le corps $\operatorname{Frac}\left(\mathcal{O}\left(\mathbf{D}_{\mathfrak{m}}^{\prime}\right)\right)$. En particulier, l'anneau $\mathscr{F}\left(\mathbf{D}_{\mathfrak{m}}^{\prime}\right)$ est intègre.

Démonstration. Notons $z_{\mathfrak{m}}$ le point 0 de la fibre $\pi^{-1}\left(\tilde{a}_{\mathfrak{m}}\right)$. D'après la discussion menée à la fin de l'annexe $\mathrm{B}$, l'anneau local en ce point est isomorphe à l'anneau $\hat{A}_{\mathfrak{m}} \llbracket T \rrbracket$. Commençons par montrer que le polynôme $Q(S)$ est irréductible sur le corps $\operatorname{Frac}\left(\mathrm{O}_{z_{\mathfrak{m}}}\right)$. Pour des raisons de valuation $T$-adique, l'élément $\pi_{\mathfrak{m}}^{n}+T$ de $\hat{A}_{\mathfrak{m}} \llbracket T \rrbracket$ n'est racine $d^{\text {ème }}$ dans $\hat{A}_{\mathfrak{m}} \llbracket T \rrbracket$ pour aucun diviseur $d \geq 2$ de $n$. D'après la théorie de Kummer, cela impose au polynôme $Q(S)$ d'être irréductible sur $\operatorname{Frac}\left(\mathrm{O}_{z_{\mathfrak{m}}}\right)$. Les mêmes arguments que dans la preuve du lemme 2.7 permettent alors de conclure.

Nous pouvons même être plus précis et démontrer un principe du prolongement analytique.

Lemme 3.4. Soient $x$ un point de $U$ et $i$ un élément de $\llbracket 1, n \rrbracket$. Le morphisme

$$
\rho_{i, x}: \mathscr{F}\left(\mathbf{D}_{\mathfrak{m}}^{\prime}\right) \rightarrow \mathscr{F}_{x} \stackrel{\varphi_{x}}{\sim} \mathbb{O}_{x}^{n} \stackrel{p_{i}}{\longrightarrow} \mathcal{O}_{x},
$$

où $p_{i}$ est la projection sur le $i^{\text {ème }}$ facteur, est injectif. 
Démonstration. Soit $s$ un élément de l'anneau $\mathscr{F}\left(\mathbf{D}_{\mathfrak{m}}^{\prime}\right)=\mathcal{O}\left(\mathbf{D}_{\mathfrak{m}}^{\prime}\right)[S] /(Q(S))$ dont l'image par le morphisme $\rho_{i, x}$ est nulle. Choisissons un élément $R(S)$ de $O\left(\mathbf{D}_{\mathfrak{m}}^{\prime}\right)[S]$ qui représente la section $s$. Reprenons les notations de la preuve de la proposition 3.2. Par hypothèse, nous avons

$$
R\left(\pi_{\mathfrak{m}} \zeta^{-i} \omega\right)=0 \text { dans } \mathscr{O}_{x} .
$$

Pour montrer que l'élément $s$ est nul, il suffit de montrer que le polynôme $Q(S)$ est le polynôme minimal de l'élément $\pi_{\mathfrak{m}} \zeta^{-i} \omega$ sur le corps $\operatorname{Frac}\left(\mathcal{O}\left(\mathbf{D}_{\mathfrak{m}}^{\prime}\right)\right)$. C'est bien le cas, puisque le lemme précédent assure que le polynôme $Q$ est irréductible sur le corps $\operatorname{Frac}\left(\mathcal{O}\left(\mathbf{D}_{\mathfrak{m}}^{\prime}\right)\right)$.

Terminons par un résultat topologique.

Lemme 3.5. La partie

$$
F=\mathbf{D}_{\mathfrak{m}}^{\prime} \backslash U=\left\{\left.x \in \mathbf{D}_{\mathfrak{m}}^{\prime}|| T(x)|\geq| \pi_{\mathfrak{m}}(x)\right|^{n}\right\}
$$

est fermée dans le disque $\mathbf{D}$.

Démonstration. Il suffit de montrer que $F$ est fermée dans $\mathbf{D}_{\mathfrak{m}}$ puisque cette dernière partie est elle-même fermée dans D. En d'autres termes, nous souhaitons montrer que la partie

$$
V=U \cup\left(\mathbf{D} \cap \pi^{-1}\left(a_{0}\right)\right)
$$

est ouverte dans $\mathbf{D}_{\mathfrak{m}}$. Puisque $U$ est une partie ouverte de $\mathbf{D}_{\mathfrak{m}}$, il suffit de montrer que $V$ est voisinage dans $\mathbf{D}_{\mathfrak{m}}$ de chacun des points de $\mathbf{D} \cap \pi^{-1}\left(a_{0}\right)$.

Soit $x$ un point de $\mathbf{D} \cap \pi^{-1}\left(a_{0}\right)$. Posons $r=|T(x)|$. C'est un élément de l'intervalle ]0,1[. Soient $s$ un élément de ] $r, 1$ [ et $\varepsilon$ un élément de ]0,1[ tels que l'on ait $\left|\pi_{\mathfrak{m}}\right|_{\mathfrak{m}}^{n \varepsilon}>s$. La partie

$$
\left\{y \in \pi ^ { - 1 } \left(\left[a_{0}, a_{\mathfrak{m}}^{\varepsilon}[)|| T(y) \mid<s\right\}\right.\right.
$$

est un voisinage ouvert du point $x$ dans $\mathbf{D}_{\mathfrak{m}}$ qui est contenu dans $V$.

3B. Raccord et retour à l'algèbre. Soit $G$ un groupe fini. Notons $n \in \mathbf{N}^{*}$ son ordre. Soient $g_{1}, \ldots, g_{t}$, avec $t \in \mathbf{N}^{*}$, des générateurs du groupe $G$. Pour tout élément $i$ de $\llbracket 1, t \rrbracket$, notons $n_{i}$ l'ordre de l'élément $g_{i}$, choisissons un nombre premier $p_{i}$ congru à 1 modulo $n_{i}$ et un idéal maximal $\mathfrak{m}_{i}$ de $A$ contenant $p_{i}$. Nous pouvons supposer que les $\mathfrak{m}_{i}$ sont distincts.

Soit $i$ un élément de $\llbracket 1, t \rrbracket$. Construisons par la méthode du numéro $3 \mathrm{~A}$ un revêtement galoisien de groupe $\mathbf{Z} / n_{i} \mathbf{Z}$. Il est défini au-dessus de $\mathbf{D}_{\mathfrak{m}_{i}}^{\prime}$ et trivial au-dessus de

$$
U_{i}=\left\{\left.x \in \mathbf{D}_{\mathfrak{m}_{i}}^{\prime}|| T(x)|<| \pi_{\mathfrak{m}_{i}}(x)\right|^{n_{i}}\right\} .
$$

Notons $\operatorname{Ind}_{\left\langle g_{i}\right\rangle}^{G}\left(\mathbf{D}_{\mathfrak{m}_{i}}^{\prime}\right)$ le $G$-revêtement induit (cf. section 1). 
D'après le lemme 3.5, pour tout élément $i$ de $\llbracket 1, t \rrbracket$, la partie $F_{i}=\mathbf{D}_{\mathfrak{m}_{i}}^{\prime} \backslash U_{i}$ est fermée dans $\mathbf{D}$. Définissons une partie ouverte de $\mathbf{D}$ par

$$
U_{0}=\mathbf{D} \backslash \bigcup_{1 \leq i \leq t} F_{i}
$$

On se convainc aisément que cet ensemble est connexe. Considérons le $G$-revêtement $\operatorname{Ind}_{\langle e\rangle}^{G}\left(U_{0}\right)$ induit par le revêtement trivial au-dessus de $U_{0}$. Recollons ces différents revêtements par la méthode décrite à la section 1 . Nous obtenons un revêtement de $\mathbf{D}$, galoisien de groupe $G$, associé à un faisceau $\mathscr{G}$. On montre à l'aide du lemme 3.4 qu'il est intègre, c'est-à-dire que l'anneau $\mathscr{G}(\mathbf{D})$ est intègre.

Nous disposons, à présent, d'un revêtement du disque $\mathbf{D}$ possédant le groupe de Galois désiré $G$. Il nous reste à montrer que l'extension induite entre les corps de fonctions est galoisienne de même groupe. Nous utiliserons, pour ce faire, le caractère Stein du disque D ( $c f$. théorème B.4).

Proposition 3.6. Le groupe des automorphismes de $\mathbf{O}(\mathbf{D})$-algèbres du faisceau $G(\mathbf{D})$ est isomorphe à $G$.

Démonstration. Soient $\mathscr{A}$ et $\mathscr{B}$ deux faisceaux de $\mathscr{O}_{\mathbf{D}}$-algèbres cohérents. Considérons l'application

$$
\operatorname{Mor}_{\mathscr{O}}(\mathscr{A}, \mathscr{B}) \rightarrow \operatorname{Mor}_{\mathscr{O}(\mathbf{D})}(\mathscr{A}(\mathbf{D}), \mathscr{B}(\mathbf{D})) .
$$

Elle est bijective car les faisceaux $\mathscr{A}$ et $\mathscr{B}$ satisfont le théorème A sur $\mathbf{D}$.

Par construction, le groupe des automorphismes de $\mathcal{O}_{\mathbf{D}}$-algèbres du faisceau $\mathscr{G}$ est isomorphe à $G$. On en déduit le résultat attendu.

Il reste à montrer que l'extension $O(\mathbf{D}) \rightarrow \mathscr{G}(\mathbf{D})$ est entière. Puisque les théorèmes du type GAGA ne sont pas valables dans ce cadre, nous utiliserons un raisonnement direct.

Lemme 3.7. Tout élément de $\mathscr{G}(\mathbf{D})$ annule un polynôme unitaire à coefficients dans $\mathbf{O}(\mathbf{D})$ de degré inférieur à $n$.

Démonstration. Soit $s$ un élément de $\mathscr{G}(\mathbf{D})$. Nous supposerons, tout d'abord, qu'il existe un point $x_{0}$ de $U_{0}$ tel que toutes les coordonnées de son image $s_{x_{0}}$ dans $\mathscr{G}_{x_{0}}=\mathrm{O}_{X, x_{0}}^{n}$ soient distinctes. Puisque l'ouvert $U_{0}$ est connexe, le principe du prolongement analytique ( $c f$. théorème B.3) assure qu'en tout point $x$ de $U_{0}$, toutes les coordonnées du germe $s_{x}$ sont distinctes. Notons $a_{1}, \ldots, a_{n}$ les coordonnées de l'image de $s$ dans $\mathscr{G}\left(U_{0}\right)=\mathrm{O}\left(U_{0}\right)^{n}$. Posons

$$
M(Z)=\prod_{l=1}^{n}\left(Z-a_{l}\right) \in \mathcal{O}\left(U_{0}\right)[Z] .
$$

En tout point $x$ de $U_{0}$, l'image du polynôme $M$ est l'unique polynôme unitaire de degré inférieur à $n$ à coefficients dans $O_{x}$ qui annule le germe $s_{x}$. 
Pour tout élément $j$ de $\llbracket 0, t \rrbracket$, posons $V_{j}=U_{0} \cup \bigcup_{1 \leq i \leq j} \mathbf{D}_{\mathfrak{m}_{i}}^{\prime}$. Montrons, par récurrence, que pour tout élément $j$ de $\llbracket 0, t \rrbracket$, il existe un polynôme unitaire $N_{j}$ de degré $n$ à coefficients dans $\mathcal{O}\left(V_{j}\right)$ qui annule l'élément $s_{\mid V_{j}}$ de $\mathscr{G}_{(}\left(V_{j}\right)$. Nous avons déjà traité le cas $j=0$. Soit maintenant un élément $j$ de $\llbracket 0, t-1 \rrbracket$ pour lequel l'hypothèse de récurrence est vérifiée. L'élément $s_{\mid \mathbf{D}_{\mathfrak{m}_{j+1}}^{\prime}}$ de l'anneau $\varphi_{n_{j+1}}\left(\mathbf{D}_{\mathfrak{m}_{j+1}}^{\prime}\right)=$ $\mathcal{O}\left(\mathbf{D}_{\mathfrak{m}_{j+1}}^{\prime}\right)[S] /\left(S^{n_{j+1}}-p_{j+1}^{n_{j+1}}-T\right)$ est annulé par un polynôme unitaire $M_{j+1}$ de degré inférieur à $n$ à coefficients dans le corps $\mathcal{O}\left(\mathbf{D}_{\mathfrak{m}_{j+1}}^{\prime}\right)$. Soit $x$ un élément de $U_{j+1}=\mathbf{D}_{\mathfrak{m}_{j+1}}^{\prime} \cap U_{0}$. Nous avons démontré qu'il existe un unique polynôme unitaire de degré inférieur à $n$ à coefficients dans $O_{x}$ qui annule le germe $s_{x}$. On en déduit que les images des polynômes $N_{j}$ et $M_{j+1}$ dans $O_{x}[Z]$ coïncident. L'ouvert $U_{j+1}$ étant connexe, d'après le théorème B.3, les images de ces polynômes dans $\mathcal{O}\left(U_{j+1}\right)[Z]$ coïncident. On en déduit que le polynôme $N_{j}$ se prolonge en un polynôme unitaire $N_{j+1}$ de degré inférieur à $n$ à coefficients dans $O\left(V_{j+1}\right)$ qui annule l'élément $s_{\mid V_{j+1}}$ de $\mathscr{G}\left(V_{j+1}\right)$.

On déduit finalement le résultat attendu du cas $j=t$.

Soit $x_{0}$ un point de l'ouvert $U_{0}$. La fibre du faisceau $\mathscr{G}$ au point $x_{0}$ est isomorphe à l'algèbre $O_{x_{0}}^{n}$. D'après le théorème $\mathrm{B} .4$, le faisceau $\mathscr{G}$ vérifie le théorème $\mathrm{A}$ de Cartan sur le disque D. On en déduit qu'il existe un élément $s_{0}$ de $\mathscr{G}(\mathbf{D})$ dont toutes les coordonnées de l'image dans la fibre $\mathscr{G}_{x_{0}}=\mathbb{O}_{x_{0}}^{n}$ sont distinctes.

Soit $s$ un élément de $\mathscr{G}(\mathbf{D})$. Il existe un élément $\lambda$ de $\mathcal{O}(\mathbf{D})$ tel que toutes les coordonnées du germe de la section $s_{1}=s+\lambda s_{0}$ au point $x_{0}$ soient distinctes. Le raisonnement qui précède montre qu'il existe deux polynômes unitaires $P_{0}$ et $P_{1}$ de degré inférieur à $n$ à coefficients dans $O(\mathbf{D})$ qui annulent respectivement les sections $s_{0}$ et $s_{1}$. On en déduit qu'il existe un polynôme unitaire $P$ de degré inférieur à $n$ à coefficients dans $\mathbf{O}(\mathbf{D})$ qui annule la section $s$.

Lemme 3.8. L'anneau A est intégralement fermé dans l'anneau $G(\mathbf{D})$.

Démonstration. Soit $P$ un polynôme unitaire à coefficients dans $A$ sans racines dans $A$. Supposons, par l'absurde, qu'il existe une section $s$ de $\mathscr{G}(\mathbf{D})$ qui est racine du polynôme $P$. Notons $z_{0}$ le point 0 de la fibre $\pi^{-1}\left(a_{0}\right)$ de l'espace $X$. C'est un point de l'ouvert $U_{0}$. Notons $a$ la première coordonnée de l'image du germe $s_{z_{0}}$ par l'isomorphisme $\mathscr{G}_{z_{0}} \simeq \mathcal{O}_{z_{0}}^{t}$. C'est un élément de $\mathcal{O}_{z_{0}}$ qui vérifie l'égalité $P(a)=0$. D'après la discussion menée à la fin de l'annexe $\mathrm{B}$, l'anneau local $\mathrm{O}_{z_{0}}$ se plonge dans l'anneau $K \llbracket T \rrbracket$. On en déduit que le polynôme $P$ possède une racine dans l'anneau $K \llbracket T \rrbracket$ et donc dans le corps $K$. Puisque l'anneau $A$ est algébriquement fermé dans le corps $K$, cette racine doit appartenir à $A$. Nous avons abouti à une contradiction. On en déduit le résultat annoncé.

Introduisons une définition correspondant à cette propriété.

Définition 3.9. Une extension $L$ du corps $\operatorname{Frac}(\mathbf{O}(\mathbf{D}))$ est dite régulière si le corps $K$ est algébriquement fermé dans $L$. 
Regroupons, à présent, les résultats obtenus.

Proposition 3.10. L'extension de corps

$$
\operatorname{Frac}(\mathbf{O}(\mathbf{D})) \rightarrow \operatorname{Frac}(\varphi(\mathbf{D}))
$$

est finie de degré $n$, régulière et galoisienne de groupe $G$.

Démonstration. L'extension Frac(O(D)) $\rightarrow$ Frac( $(G(D))$ est finie et de degré inférieur à $n$ d'après le lemme 3.7. Elle est régulière d'après le lemme 3.8. On déduit de la proposition 3.6 qu'il existe un morphisme injectif du groupe $G$ dans le groupe des $\operatorname{Frac}(\mathcal{O}(\mathbf{D}))$-automorphismes du corps $\operatorname{Frac}(\mathscr{G}(\mathbf{D}))$. Or le groupe $G$ a pour cardinal $n$. On en déduit que l'extension Frac(O(D)) $\rightarrow$ Frac( $(\mathscr{G}(\mathbf{D}))$ est exactement de degré $n$, qu'elle est galoisienne et que son groupe de Galois est isomorphe au groupe $G$.

Remarque 3.11. Puisque le disque $\mathbf{D}$ est connexe, les théorèmes B.3 et B.4 assurent que l'anneau des sections méromorphes globales $M(\mathbf{D})$ est un corps isomorphe à $\operatorname{Frac}(\mathbf{O}(\mathbf{D}))$. L'extension Frac(O(D)) $\rightarrow$ Frac( $(\mathscr{G}(\mathbf{D}))$ est donc bien l'extension obtenue à partir du revêtement du disque $\mathbf{D}$ associé au faisceau $\mathscr{G}$ en passant aux corps de fonctions.

3C. Conclusion et généralisations. Regroupons, à présent, les résultats que nous avons obtenus. Puisque nous sommes partis d'un groupe fini $G$ arbitraire, nous avons finalement démontré que tout groupe fini est groupe de Galois d'une extension finie et régulière du corps $\operatorname{Frac}(\mathbf{O}(\mathbf{D}))$. D' après la description de l'anneau $\mathbf{O}(\mathbf{D})$ donnée à la fin de l'annexe $\mathrm{B}$, ce dernier est isomorphe au corps $\operatorname{Frac}\left(A_{1^{-}} \llbracket T \rrbracket\right)$, où $A_{1^{-}} \llbracket T \rrbracket$ désigne l'anneau des séries en une variable à coefficients dans $A$ de rayon de convergence complexe supérieur ou égal à 1 en toute place infinie. Lorsque $A=\mathbf{Z}$, nous retrouvons bien ainsi le résultat de Harbater [1988, Corollary 3.8] énoncé en introduction.

Théorème 3.12. Soit A un anneau d'entiers de corps de nombres. Tout groupe fini est groupe de Galois d'une extension finie et régulière du corps $\operatorname{Frac}\left(A_{1^{-}} \llbracket T \rrbracket\right)$.

Remarque 3.13. Pour tout $r>1$, l'anneau $A_{r}-\llbracket T \rrbracket$ des séries en une variable à coefficients dans $A$ de rayon de convergence complexe supérieur ou égal à $r$ en toute place infinie (une seule suffirait) est réduit à l'anneau de polynômes $A[T]$. Si nous disposions du théorème précédent pour un certain nombre réel $r>1$, nous aurions donc résolu le problème inverse de Galois géométrique sur $K$.

Pour finir, nous regroupons plusieurs résultats proches de celui du théorème 3.12. Les démonstrations en sont fort similaires et nous n'indiquerons que les modifications à effectuer.

Ainsi que nous l'avons déjà signalé, le spectre analytique d'un anneau d'entiers de corps de nombres présente de nombreuses similitudes avec la droite analytique 
sur un corps trivialement valué. C'est donc, tout d'abord, dans ce cadre que nous allons nous placer. Soit $k$ un corps. Munissons-le de la valeur absolue triviale afin d'en faire un corps ultramétrique complet. Considérons, maintenant, l'espace $\mathbf{A}_{k}^{2 \text {, an }}$, analogue de $\mathbf{A}_{A}^{1, \text { an }}$. Nous noterons $U$ et $T$ les coordonnées sur cet espace.

Lorsque la caractéristique du corps $k$ est nulle, pour tout entier $n \geq 1$, il existe une infinité de branches de la droite $\mathbf{A}_{k}^{1 \text {,an }}$ dont l'anneau des fonctions contient une racine primitive $n^{\text {ème }}$ de l'unité. Posons

$$
\mathbf{D}_{k}=\left\{x \in \mathbf{A}_{k}^{2, \text { an }}|| T(x) \mid<1\right\} .
$$

En appliquant le raisonnement de cette section, nous démontrons que tout groupe fini est groupe de Galois d'une extension finie et régulière du corps $\operatorname{Frac}\left(\mathcal{O}\left(\mathbf{D}_{k}\right)\right)$. Par régulière, nous entendons ici que le corps $k(U)$ est algébriquement fermé dans l'extension en question.

Supposons, à présent, que le corps $k$ est de caractéristique $p$, où $p$ est un nombre premier. Dans ce cas, la construction des revêtements cycliques locaux est plus complexe. Cependant, il est possible de la mener à bien en faisant appel aux extensions d'Artin-Schreier-Witt, comme nous l'avons déjà fait au numéro $2 \mathrm{~A}$. Il est plus difficile de montrer qu'un tel revêtement est trivial sur une partie dont le complémentaire est fermé dans $\mathbf{D}_{k}$, mais les propriétés du flot nous permettent d'y parvenir.

En utilisant une description explicite de l'anneau $\mathcal{O}\left(\mathbf{D}_{k}\right)$, nous obtenons finalement le résultat suivant :

Théorème 3.14. Soit $k$ un corps. Notons

$$
k_{+\infty, 1^{-} \llbracket U, T \rrbracket}
$$

le sous-anneau de $k[U] \llbracket T \rrbracket$ composé des séries de la forme

$$
\sum_{n \geq 0} a_{n}(U) T^{n}
$$

qui vérifient la condition

$$
\forall r>0, \forall s \in\left[0,1\left[, \lim _{n \rightarrow+\infty}\left(r^{\operatorname{deg}\left(a_{n}\right)} s^{n}\right)=0 .\right.\right.
$$

Tout groupe fini est groupe de Galois d'une extension finie et régulière du corps $\operatorname{Frac}\left(k_{+\infty, 1^{-}} \llbracket U, T \rrbracket\right)$.

Remarque 3.15. Le théorème précédent nous permet, en particulier, de réaliser, pour tout corps $k$, tout groupe fini comme groupe de Galois sur le corps des fractions de $k[U] \llbracket T \rrbracket$. Nous étendons ainsi les corollaires 1.4 et 1.5 de [Harbater 1984b]. 


\section{Annexe A. Théorèmes GAGA relatifs sur un affinoïde}

Soit $k$ un corps muni d'une valeur absolue ultramétrique pour laquelle il est complet, $\mathscr{A}$ une algèbre $k$-affinoïde et $X$ un schéma localement de type fini sur $\mathscr{A}$. Berkovich [1993, 2.6] a défini, de manière fonctorielle, l'analytifié $X^{\text {an }}$ du schéma $X$. Il vient avec un morphisme d'espaces localement annelés $X^{\text {an }} \rightarrow X$, qui est plat et surjectif (cette dernière propriété tombe évidemment en défaut dans le cas complexe). À tout faisceau de $\mathcal{O}_{X}$-modules $\mathscr{F}_{\text {}}$, nous pouvons associer, par rétrotirette, un faisceau de $\widehat{O}_{X^{\text {an }}}$-modules, que nous noterons $\mathscr{F}^{\text {an }}$. Remarquons que l'analytifié d'un faisceau cohérent est encore cohérent.

Dans la lignée des théorèmes GAGA [Serre 1955-1956; SGA1 1971, exposé XII] nous allons nous intéresser aux propriétés du foncteur d'analytification lorsque l'espace $X$ est propre. Précisément, nous allons démontrer le théorème suivant :

Théorème A.1. Soit $k$ un corps muni d'une valeur absolue ultramétrique pour laquelle il est complet, $\mathscr{A}$ une algèbre $k$-affinoüde et $X$ un $\mathscr{A}$-schéma propre.

(i) Pour tout faisceau de $\mathbb{O}_{X}$-modules cohérent $\mathscr{F}_{F}$ et tout entier $q \in \mathbf{N}$, le morphisme $H^{q}(X, \mathscr{F}) \rightarrow H^{q}\left(X^{\text {an }}, \mathscr{F}^{\text {an }}\right)$ est un isomorphisme.

(ii) Le foncteur d'analytification $\mathscr{F}^{\rightarrow} \mathscr{F}^{\mathrm{an}}$ induit une équivalence entre la catégorie des $\mathrm{O}_{X}$-modules cohérents et celle des $\mathbb{O}_{X}$ an-modules cohérents.

La preuve originale de Serre, qui concerne l'analytification complexe, peut être adaptée à notre contexte sans difficultés majeures. Signalons que le théorème précédent a d'ailleurs déjà été obtenu par U. Köpf [1974] dans le cadre de la géométrie rigide et par A. Ducros en général, dans un texte inédit. Nous en rédigeons cependant une démonstration pour la commodité du lecteur, sans prétendre aucunement à l'originalité.

Comme dans le cas complexe, on se ramène à démontrer le théorème pour un espace $X$ de la forme $\mathbf{P}_{\mathscr{A}}^{r}$ et on utilise les résultats classiques concernant les faisceaux cohérents sur un tel espace. Deux propriétés joueront un rôle essentiel dans la preuve : la platitude du morphisme $X^{\text {an }} \rightarrow X$ et la finitude cohomologique des morphismes propres. Ce dernier point prend la forme du théorème suivant :

Théorème A.2. Soit $k$ un corps muni d'une valeur absolue ultramétrique pour laquelle il est complet, $\mathscr{A}$ une algèbre $k$-affinoüde et $X$ un $\mathscr{A}$-espace analytique propre. Pour tout faisceau de $\mathrm{O}_{X}$-modules cohérent et tout entier naturel $q$, le $A$ module $H^{q}(X, \mathscr{F})$ est un $\mathscr{A}$-module de Banach de type fini.

Ce résultat a été démontré par R. Kiehl [1967, Theorem 3.3] dans le cas d'un corps de valuation non triviale et d'objets strictement affinoïdes. Il a été étendu au cas général par Berkovich [1990, Proposition 3.3.5]. 
Indiquons pour finir le seul véritable ajout que nous avons dû faire à la preuve de Serre (et qui figure chez Ducros) : il s'agit du lemme A.4, un résultat de changement de base, utilisé pour pallier le fait qu'un point de l'espace $k$-analytique $\mathbf{P}_{k}^{r \text {,an }}$ n'est pas toujours situé sur un hyperplan.

Démonstration du théorème A.1. Commençons par quelques réductions classiques. En utilisant le lemme de Chow [EGA II 1961, théorème 5.6.1], on montre qu'il suffit de prouver le théorème dans le cas où $X$ est un schéma projectif sur $\mathscr{A}$. Dans le cas complexe, les détails de l'argument figurent dans [SGA1 1971, exposé XII] ; un raisonnement en tout point semblable vaut ici.

Si $X$ est un schéma projectif sur $\mathscr{A}$, il existe une immersion fermée $\varphi: X \rightarrow \mathbf{P}_{\mathscr{A}}^{r}$, pour un certain entier naturel $r$. Pour tout faisceau de $\mathscr{O}_{X}$-modules cohérent $\mathscr{F}_{\text {, }}$ le faisceau de $\mathscr{O}_{\mathbf{P}^{r}}$-modules $\varphi_{*}\left(\mathscr{F}_{F}\right)$, qui n'est autre que le prolongement de $\mathscr{F}_{F}$ par zéro, est encore cohérent. On vérifie que cette opération de prolongement commute à l'analytification et préserve la cohomologie. En outre, elle possède un inverse à gauche : la restriction à $Y$. En utilisant ces propriétés, on montre qu'il suffit de prouver le théorème dans le cas où $X$ est un espace projectif sur $\mathscr{A}$. C'est ce que nous supposerons désormais.

Assertion (i) lorsque $\mathscr{F}=\mathscr{O}(n)$. Nous allons démontrer, par récurrence sur $r$, que, pour tout entier naturel $r$ et tout entier relatif $n$, l'assertion (i) du théorème est vraie lorsque $X=\mathbf{P}_{\mathscr{A}}^{r}$ et $\mathscr{F}_{F}=\mathrm{O}_{X}(n)$.

Pour $r=0$, le résultat découle du théorème d'acyclicité de Tate.

Soit $r \in \mathbf{N}$ tel que le résultat soit vrai pour $\mathbf{P}_{\mathscr{A}}^{r}$. Posons $X=\mathbf{P}_{\mathscr{A}}^{r+1}$. Soit $t$ une section non nulle du fibré $\mathfrak{O}_{X}(1)$ et $Y$ l'hyperplan de $X$ (isomorphe à $\mathbf{P}_{\mathscr{A}}^{r}$ ) qu'elle définit. Nous noterons $\mathscr{O}_{Y}$ à la fois le faisceau structural sur $Y$ et son prolongement par zéro à $X$. D'après l'hypothèse de récurrence, pour tout entier $q \in \mathbf{N}$, le morphisme

$$
H^{q}\left(X, \mathscr{O}_{Y}(n)\right)=H^{q}\left(Y, \mathscr{O}_{Y}(n)\right) \rightarrow H^{q}\left(Y^{\mathrm{an}}, \mathscr{O}_{Y} \text { an }(n)\right)=H^{q}\left(X^{\mathrm{an}}, \mathrm{O}_{Y} \text { an }(n)\right)
$$

est un isomorphisme.

Pour tout $n \in \mathbf{Z}$, la multiplication par $t$ définit une suite exacte courte

$$
0 \rightarrow \mathrm{O}_{X}(n-1) \rightarrow \mathrm{O}_{X}(n) \rightarrow \mathrm{O}_{Y}(n) \rightarrow 0 .
$$

En écrivant la suite exacte longue associée et en utilisant le lemme des cinq, on montre que l'on a un isomorphisme

$$
H^{q}\left(X, \mathrm{O}_{X}(n)\right) \simeq H^{q}\left(X^{\text {an }}, \mathrm{O}_{X^{\text {an }}}(n)\right)
$$

pour tout $q \in \mathbf{N}$ si, et seulement si, on a un isomorphisme

$$
H^{q}\left(X, \mathscr{O}_{X}(n-1)\right) \simeq H^{q}\left(X^{\text {an }}, \mathscr{O}_{X^{\text {an }}}(n-1)\right)
$$

pour tout $q \in \mathbf{N}$. 
Un calcul explicite montre que l'on a $H^{q}\left(X, \mathcal{O}_{X}\right) \simeq H^{q}\left(X^{\text {an }}, \mathcal{O}_{X^{\text {an }}}\right)$ pour tout $q \in \mathbf{N}$. On en déduit le résultat annoncé.

Assertion (i) en général. Soit $r \in \mathbf{N}$. Posons $X=\mathbf{P}_{\mathscr{A}}^{r}$. Nous allons démontrer, par une récurrence descendante sur $q$, que, pour tout entier naturel $q$, l'assertion (i) du théorème est vraie pour $H^{q}$.

Si $q>r$, pour tout faisceau de $\mathscr{O}_{X}$-modules cohérent $\mathscr{F}$, les groupes $H^{q}(X, \mathscr{F})$ et $H^{q}\left(X^{\text {an }}, \mathscr{F}^{\text {an }}\right)$ sont tous deux nuls, et le résultat est vrai.

Soit $q \in \mathbf{N}^{*}$ tel que le résultat soit vrai pour $H^{q}$. Soit $\mathscr{F}$ un faisceau de $\mathrm{O}_{X^{-}}$ modules cohérent. Nous pouvons l'insérer dans une suite exacte de faisceaux de $\mathrm{O}_{X}$-modules cohérents

$$
0 \rightarrow \mathscr{R} \rightarrow \mathscr{L} \rightarrow \mathscr{F} \rightarrow 0,
$$

où $\mathscr{L}$ est somme directe de faisceaux isomorphes à $\mathcal{O}(n)$, avec $n \in \mathbf{Z}$.

Insérons le faisceau $\mathscr{R}$ dans une suite exacte courte du même type

$$
0 \rightarrow \mathscr{R}^{\prime} \rightarrow \mathscr{L}^{\prime} \rightarrow \mathscr{R} \rightarrow 0 .
$$

D'après l'hypothèse de récurrence et le cas $\mathscr{F}=\mathscr{O}(n)$ déjà démontré, le résultat vaut pour le faisceau $\mathscr{R}^{\prime}$ en rang $q$ et pour le faisceau $\mathscr{L}^{\prime}$ en rangs $q$ et $q-1$. Le lemme des cinq assure alors que le morphisme

$$
H^{q-1}(X, \mathscr{R}) \rightarrow H^{q-1}\left(X^{\text {an }}, \mathscr{R}^{\text {an }}\right)
$$

est surjectif.

Pour les mêmes raisons que précédemment, nous savons en outre que le résultat vaut pour le faisceau $\mathscr{R}$ en rang $q$ et pour le faisceau $\mathscr{L}$ en rangs $q$ et $q-1$. Une nouvelle application du lemme des cinq assure alors que le morphisme

$$
H^{q-1}(X, \mathscr{F}) \rightarrow H^{q-1}\left(X^{\text {an }}, \mathscr{F}^{\text {an }}\right)
$$

est bijectif.

Pleine fidélité $d u$ foncteur $\mathscr{F}_{F} \rightarrow \mathscr{F}^{\text {an }}$. Soit $X$ un espace projectif sur $\mathscr{A}$. Soient $\mathscr{F}$ et $\mathscr{G}$ deux faisceaux de $\mathscr{O}_{X}$-modules cohérents. Soit $x^{\text {an }}$ un point de $X^{\text {an }}$. Notons $x$ son image dans $X$.

Nous disposons des isomorphismes

et

$$
\mathscr{H} o m(\mathscr{F}, G)_{x^{\mathrm{an}}}^{\mathrm{an}} \simeq \operatorname{Hom}(\mathscr{F}, \mathscr{G})_{x} \otimes_{\mathbb{O}_{X, x}} \mathcal{O}_{X}^{\mathrm{an}}, x^{\text {an }}
$$

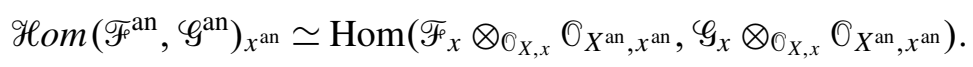

La platitude du morphisme naturel $X^{\text {an }} \rightarrow X$ entraîne que le morphisme

$$
\mathscr{H o m}(\mathscr{F}, \mathscr{G})^{\text {an }} \rightarrow \mathscr{H} \operatorname{Hom}\left(\mathscr{F}^{\text {an }}, \mathscr{G}^{\text {an }}\right)
$$

est un isomorphisme. 
On conclut en appliquant le résultat de l'assertion (i) du théorème au faisceau cohérent $\mathscr{H o m}(\mathscr{F}, \mathscr{G})$ et à l'entier $q=0$.

Surjectivité essentielle du foncteur $\mathscr{F} \rightarrow \mathscr{F}^{\text {an }}$. Nous allons démontrer, par récurrence sur $r$, que, pour tout entier naturel $r$, le foncteur $\mathscr{F}_{\rightarrow} \rightarrow \mathscr{F}^{\text {an }}$ est essentiellement surjectif lorsque $X=\mathbf{P}_{\mathscr{A}}^{r}$.

Lorsque $r=0$, le résultat est classique [Berkovich 1990, Proposition 2.3.1].

Soit $r \in \mathbf{N}$ tel que le résultat soit vrai pour le schéma $\mathbf{P}_{\mathscr{A}}^{r}$. Posons $X=\mathbf{P}_{\mathscr{A}}^{r+1}$. Commençons par une série de lemmes.

Lemme A.3. Pour chaque hyperplan projectif $Y$ de $X$ et chaque faisceau de $O_{X^{\text {an }}}$ modules cohérent $\mathcal{N}$, il existe un entier $n_{0}$ tel que

$$
\forall n \geq n_{0}, \forall q \geq 1, H^{q}\left(Y^{\text {an }}, \mathcal{N}_{\mid Y^{\text {an }}}(n)\right)=0 .
$$

Démonstration. On démontre ce résultat en appliquant l'hypothèse de récurrence au faisceau $\mathcal{N}_{\mid Y \text { an }}$, puis en utilisant le résultat analogue pour les schémas projectifs et les isomorphismes fournis par l'assertion (i) du théorème.

Lemme A.4. Soit L une extension valuée complète de $k$. Notons $\pi: X_{L}^{\mathrm{an}} \rightarrow X^{\mathrm{an}}$ le morphisme de projection. Soient $x$ un point de $X^{\text {an }}$ et $x_{L}$ l'un de ses antécédents par le morphisme $\pi$. Soit $\mathscr{F}$ un faisceau de $\mathbb{O}_{X}$ an-modules cohérent. Supposons que la fibre $\pi^{*}(\mathscr{F})_{x_{L}}$ soit engendrée par l'ensemble des sections globales de $\pi^{*}(\mathscr{F})$. Alors, la fibre $\mathscr{F}_{x}$ est engendrée par l'ensemble des sections globales de $\mathscr{F}$.

Démonstration. D'après le théorème A.2, $\mathscr{F}\left(X^{\text {an }}\right)$ est un $\mathscr{A}$-module de Banach fini. Considérons une famille $\left(f_{1}, \ldots, f_{r}\right)$, avec $r \in \mathbf{N}$, qui l'engendre. Notons $\varphi$ le conoyau du morphisme $\mathbb{O}_{X^{\text {an }}}^{r} \rightarrow \mathscr{F}$ défini par cette famille. Puisque le produit tensoriel est exact à droite, le faisceau $\pi^{*}(\mathscr{G})$ est le conoyau du morphisme $\mathcal{O}_{X_{L}^{\text {an }}}^{r} \rightarrow \pi^{*}(\mathscr{F})$ défini par la famille $\left(\pi^{*}\left(f_{1}\right), \ldots, \pi^{*}\left(f_{r}\right)\right)$.

L'exactitude du foncteur $\cdot \hat{\otimes}_{k} L$ assure que les $\left(\mathscr{A} \hat{\otimes}_{k} L\right)$-modules $\pi^{*}(\mathscr{F})\left(X_{L}^{\mathrm{an}}\right)$ et $\mathscr{F}\left(X^{\mathrm{an}}\right) \hat{\otimes}_{k} L$ sont isomorphes. En particulier, la famille $\left(\pi^{*}\left(f_{1}\right), \ldots, \pi^{*}\left(f_{r}\right)\right)$ engendre $\pi^{*}(\mathscr{F})\left(X_{L}^{\text {an }}\right)$. Puisque, par hypothèse, cet ensemble engendre $\pi^{*}(\mathscr{F})_{X_{L}}$, la fibre $\pi^{*}\left(\mathscr{G}_{x_{L}} \simeq \mathscr{G}_{x} \otimes_{\mathcal{O}_{X}^{\mathrm{an}, x}} \mathcal{O}_{X_{L}^{\mathrm{an}}, x_{L}}\right.$ est nulle. A fortiori, nous avons $\mathscr{G}_{x} \otimes_{\mathcal{O}_{X} \mathrm{an}, x}$ $\kappa\left(x_{L}\right)=0$. Puisque $\mathscr{G}_{x} \otimes_{\mathcal{O}_{X} \text { an }, x} \kappa\left(x_{L}\right) \simeq \mathscr{G}_{x} \otimes_{\mathbb{O}_{X \text { an }, x}} \kappa(x) \otimes_{\kappa(x)} \kappa\left(x_{L}\right)$, nous avons même $\mathscr{G}_{x} \otimes_{\mathrm{O}_{\mathrm{X}}^{\mathrm{an}, x}} \kappa(x)=0$, d'où l'on déduit que $\mathscr{G}_{x}=0$, par le lemme de Nakayama.

Lemme A.5. Soient $\mathcal{N}$ un faisceau de $\mathcal{O}_{X^{\text {an }}}$-modules cohérent et $x$ un point de $X^{\mathrm{an}}$. Il existe un entier naturel $n_{0}$ tel que, pour tout $n \geq n_{0}$, la fibre $\mathcal{N}(n)_{x}$ soit engendrée par l'ensemble des sections globales de $\mathcal{N}(n)$.

Démonstration. D'après le lemme A.4, quitte à effectuer un changement de corps de base de $k$ à $\mathscr{H}(x)$ (et à modifier les autres données en conséquence), nous pouvons supposer que le point $x$ est $k$-rationnel. Il est alors situé sur l'analytifié $Y^{\text {an }}$ d'un certain hyperplan projectif $Y$ de $X$. 
Soit $t$ une section de $\mathscr{O}_{X}(1)$ de lieu des zéros $Y$. La multiplication par $t$ définit une suite exacte

$$
0 \rightarrow \mathcal{N}^{\prime} \rightarrow \mathcal{N}(-1) \rightarrow \mathcal{N} \rightarrow \mathcal{N}_{\mid Y^{\text {an }}} \rightarrow 0,
$$

où $\mathcal{N}^{\prime}$ est un faisceau de $\mathbb{O}_{X}$ an-modules cohérent supporté par $Y^{\text {an }}$.

Soit $n \in \mathbf{Z}$. En tensorisant la suite précédente par $\mathcal{O}_{X^{\text {an }}}(n)$ puis en la scindant, nous obtenons les deux suites exactes courtes

$$
0 \rightarrow \mathcal{N}^{\prime}(n) \rightarrow \mathcal{N}(n-1) \rightarrow \mathscr{P}_{n} \rightarrow 0
$$

et

$$
0 \rightarrow \mathscr{P}_{n} \rightarrow \mathcal{N}(n) \rightarrow \mathcal{N}_{\mid Y \text { an }}(n) \rightarrow 0,
$$

qui donnent naissance aux deux suites exactes de cohomologie

$$
H^{1}\left(X^{\mathrm{an}}, \mathcal{N}(n-1)\right) \rightarrow H^{1}\left(X^{\mathrm{an}}, \mathscr{P}(n)\right) \rightarrow H^{2}\left(X^{\mathrm{an}}, \mathcal{N}^{\prime}(n)\right)
$$

et

$$
H^{1}\left(X^{\text {an }}, \mathscr{P}_{n}\right) \rightarrow H^{1}\left(X^{\text {an }}, \mathcal{N}(n)\right) \rightarrow H^{1}\left(X^{\text {an }}, \mathcal{N}_{\mid Y^{\text {an }}}(n)\right) .
$$

D'après le lemme A.3, il existe un entier $n_{1}$ tel que, pour tout $n \geq n_{1}$, les groupes de cohomologie

$$
H^{2}\left(X^{\text {an }}, \mathcal{N}^{\prime}(n)\right) \text { et } H^{1}\left(X^{\text {an }}, \mathcal{N}_{\mid Y \text { an }}(n)\right)
$$

soient nuls et, par conséquent, le morphisme composé

$$
H^{1}\left(X^{\text {an }}, \mathcal{N}(n-1)\right) \rightarrow H^{1}\left(X^{\text {an }}, \mathscr{P}_{n}\right) \rightarrow H^{1}\left(X^{\text {an }}, \mathcal{N}(n)\right)
$$

soit surjectif.

Le morphisme $X^{\text {an }} \rightarrow M(\mathscr{A})$ étant propre, le théorème A.2, assure que le $\mathscr{A}$ module $H^{1}\left(X^{\text {an }}, \mathcal{N}\left(n_{1}-1\right)\right)$ est de type fini, et donc noethérien. On en déduit qu'il existe un entier $n_{2} \geq n_{1}$ tel que, pour tout $n \geq n_{2}$, le morphisme

$$
H^{1}\left(X^{\mathrm{an}}, \mathcal{N}(n-1)\right) \rightarrow H^{1}\left(X^{\mathrm{an}}, \mathcal{N}(n)\right)
$$

soit un isomorphisme. Par conséquent, pour tout $n \geq n_{2}$, le morphisme surjectif

$$
H^{1}\left(X^{\text {an }}, \mathscr{P}_{n}\right) \rightarrow H^{1}\left(X^{\text {an }}, \mathcal{N}(n)\right)
$$

est bijectif, d'où l'on déduit, en considérant la suite exacte longue associé à la seconde suite exacte courte, que le morphisme

$$
H^{0}\left(X^{\text {an }}, \mathcal{N}(n)\right) \rightarrow H^{0}\left(X^{\text {an }}, \mathcal{N}_{\mid Y^{\text {an }}}(n)\right)
$$

est surjectif.

D'après l'hypothèse de récurrence, le faisceau de $\mathscr{O}_{Y}$ an -modules cohérent $\mathcal{N}_{\mid Y^{\text {an }}}$ est l'analytifié d'un faisceau de $\mathfrak{O}_{Y}$-modules cohérent $\mathscr{G}$. Notons $x^{\text {alg }}$ l'image du point $x$ dans $Y$. Les résultats classiques sur les schémas projectifs assurent qu'il existe un entier $n_{0} \geq n_{2}$ tel que, pour tout $n \geq n_{0}$, la fibre $\mathscr{G}(n)_{x}$ alg soit engendrée, 



utilisant l'assertion (i) du théorème, on en déduit que le résultat vaut encore en remplaçant respectivement $Y$ par $Y^{\text {an }}, \mathscr{G}$ par $\mathcal{N}_{\mid Y^{\text {an }}}$ et $x^{\text {alg }}$ par $x$.

Notons $\mathscr{I}$ le faisceau d'idéaux qui définit $Y^{\text {an }}$ dans $X^{\text {an }}$. Remarquons que sa fibre $\mathscr{I}_{x}$ est contenue dans l'idéal maximal $\mathfrak{m}_{x}$ de $\mathscr{O}_{X^{\text {an }}, x}$. Soit $n \geq n_{0}$. Nous venons de montrer que $\mathcal{N}(n)_{x} \otimes_{\mathscr{O}_{X} \text { an }, x}\left(\mathcal{O}_{X^{\text {an }}, x} / \mathscr{I}_{X}\right)$ est engendré par $H^{0}\left(X^{\text {an }}, \mathcal{N}_{\mid Y^{\text {an }}}(n)\right)$. On en déduit que $\mathcal{N}(n)_{x} \otimes_{\mathcal{O}_{X} \text { an }, x}\left(\mathcal{O}_{X^{\text {an }}, x} / \mathfrak{m}_{x}\right)$ est engendré par $H^{0}\left(X^{\text {an }}, \mathcal{N}_{\mid Y \text { an }}(n)\right)$, et donc par $H^{0}\left(X^{\text {an }}, \mathcal{N}(n)\right)$. On conclut par le lemme de Nakayama.

Terminons, à présent, la démonstration du théorème A.1. Soit $\mathcal{N}$ un faisceau de $O_{X}$ an-modules cohérent. En utilisant le résultat du lemme précédent et la compacité de $X^{\text {an }}$, on montre qu'il existe un entier $n$ tel qu'en tout point $x$ de $X^{\text {an }}$, la fibre $\mathcal{N}(n)_{x}$ soit engendrée par $H^{0}\left(X^{\text {an }}, \mathcal{N}(n)\right)$. On en déduit l'existence d'un entier naturel $p$, d'un faisceau de $\mathcal{O}_{X}$ an-modules cohérent $\mathscr{R}$ et d'une suite exacte $0 \rightarrow \mathscr{R} \rightarrow \mathcal{O}_{X^{\text {an }}}(-n)^{p} \rightarrow \mathcal{N} \rightarrow 0$. En appliquant le même raisonnement au faisceau $\mathscr{R}$, nous parvenons finalement à écrire le faisceau $\mathcal{N}$ comme le conoyau d'un morphisme $\varphi: \mathbb{O}_{X^{\text {an }}}(-m)^{q} \rightarrow \mathcal{O}_{X^{\text {an }}}(-n)^{p}$, avec $m \in \mathbf{Z}$ et $q \in \mathbf{N}$. Puisque le foncteur d'analytification est pleinement fidèle, le morphisme $\varphi$ est l'analytifié d'un morphisme $\varphi^{\text {alg }}: \mathrm{O}_{X}(-m)^{q} \rightarrow \mathrm{O}_{X}(-n)^{p}$. L'exactitude à droite du foncteur d'analytification assure alors que le faisceau $\mathcal{N}$ est isomorphe à l'analytifié du conoyau du morphisme $\varphi^{\text {alg }}$, qui est un faisceau de $\mathscr{O}_{X}$-modules cohérent.

Corollaires. Nous énonçons ici deux corollaires du théorème A.1. Ils ont également pour objet des résultats de type GAGA, mais sur des bases qui ne sont plus nécessairement affinoïdes. Nous sommes convaincu qu'il est possible de les étendre à une base quelconque, de façon à obtenir un analogue parfait des théorèmes obtenus par M. Hakim [1972, chapitre VIII, théorèmes 3.2 et 3.5] dans le cadre de la géométrie analytique complexe. Cependant, pour éviter d'avoir à utiliser le formalisme un peu lourd des schémas relatifs sur un espace analytique, nous nous contenterons d'énoncer les deux cas particuliers que nous utilisons dans cet article.

Soit $k$ un corps muni d'une valeur absolue ultramétrique pour laquelle il est complet, $\mathscr{A}$ une algèbre $k$-affinoïde et $X$ un $\mathscr{A}$-schéma propre. Soit $B$ une partie compacte de $M(\mathscr{A})$ possédant un système fondamental de voisinages affinoïdes. Rappelons que la notation $O(B)$ désigne l'anneau des germes de fonctions analytiques au voisinage de $B$. Notons $Y=X \times \operatorname{Spec}(\mathscr{A}) \operatorname{Spec}(O(B))$ et désignons par $Y^{\text {an }}$ l'image réciproque de $B$ dans $X^{\text {an }}$. Munissons $Y^{\text {an }}$ du faisceau des fonctions surconvergentes. En utilisant le morphisme d'analytification au-dessus d'un espace affinoïde défini par V. Berkovich, on montre qu'il existe un morphisme d'espaces localement annelés $Y^{\text {an }} \rightarrow Y$. La rétrotirette d'un faisceau de $\mathcal{O}_{Y}$-modules cohérent $\mathscr{F}_{F}$ est un faisceau de $\mathrm{O}_{Y^{\text {an }}}$-modules cohérent, que nous noterons $\mathscr{F}^{\mathrm{an}}$. 
Corollaire A.6. Supposons que nous nous trouvons dans la situation décrite cidessus.

(i) Pour tout faisceau de $\mathbb{O}_{Y}$-modules cohérent $\mathscr{F}$ et tout entier $q \in \mathbf{N}$, le morphisme $H^{q}(Y, \mathscr{F}) \rightarrow H^{q}\left(Y^{\text {an }}\right.$, F्Fan $)$ est un isomorphisme.

(ii) Le foncteur d'analytification $\mathscr{F}^{\rightarrow} \mathscr{F}^{\mathrm{an}}$ induit une équivalence entre la catégorie des $\mathrm{O}_{Y}$-modules cohérents et celle des $\mathrm{O}_{Y}$ an-modules cohérents.

Démonstration. Il faut tout d'abord remarquer que l'espace $Y^{\text {an }}$ est compact. En reprenant le raisonnement de la preuve de la proposition 1 de [Cartan 1951], on en déduit que tout faisceau cohérent sur $Y^{\text {an }}$ se prolonge en un faisceau cohérent sur un voisinage de $Y^{\text {an }}$, et donc sur une partie de la forme $Y^{\text {an }} \times_{\mathcal{M}(\mathscr{A})} V$, où $V$ est un voisinage affinoïde de $B$. En utilisant ce raisonnement et le théorème A.1, on obtient le résultat attendu.

Soit $k$ un corps muni d'une valeur absolue ultramétrique pour laquelle il est complet, $\mathscr{A}$ une algèbre $k$-affinoïde et $X$ un $\mathscr{A}$-schéma propre. Soit $B$ un espace $A$-analytique qui soit limite inductive d'espaces affinoïdes. Notons $Z=X \times \operatorname{Spec}(\mathscr{A})$ $\operatorname{Spec}(O(B))$. En utilisant le morphisme d'analytification au-dessus d'un espace affinoïde défini par Berkovich, on construit, par limite inductive, un espace analytique $Z^{\text {an }}$ et un morphisme d'espaces localement annelés $Z^{\text {an }} \rightarrow Z$. Comme précédemment, la rétrotirette d'un faisceau de $0_{Z}$-modules cohérent $\mathscr{F}$ est un faisceau de $\mathrm{O}_{Z}$ an -modules cohérent, que nous noterons $\mathscr{F}^{\text {an }}$. Le résultat suivant se déduit alors aisément du théorème A.1.

Corollaire A.7. Supposons que nous nous trouvons dans la situation décrite cidessus.

(i) Pour tout faisceau de $\mathrm{O}_{Z}$-modules cohérent $\mathscr{F}$ et tout entier $q \in \mathbf{N}$, le morphisme $H^{q}(Z, \mathscr{F}) \rightarrow H^{q}\left(Z^{\text {an }}, \mathscr{F}^{\text {an }}\right)$ est un isomorphisme.

(ii) Le foncteur d'analytification $\mathscr{F}^{\rightarrow} \mathscr{F}^{\mathrm{an}}$ induit une équivalence entre la catégorie des $\mathrm{O}_{Z}$-modules cohérents et celle des $\mathrm{O}_{Z}$ an-modules cohérents.

\section{Annexe B. La droite de Berkovich sur un anneau d'entiers de corps de nombres}

Dans cette annexe, nous présentons succintement la droite de Berkovich sur un anneau d'entiers de corps de nombres. Nous invitons le lecteur dont ces prémices auront éveillé la curiosité à parcourir l'ouvrage [Poineau 2008] pour approfondir ce sujet.

Définitions. Soit $K$ un corps de nombres. Notons $A$ l'anneau de ses entiers. Commençons par rappeler la définition d'espace affine analytique sur $A$. Elle est due à Berkovich [1990, §1.5]. Soit $n \in \mathbf{N}$. L'espace affine analytique de dimension $n$ 
sur $A$, noté $\mathbf{A}_{A}^{n \text {,an }}$, est l'ensemble des semi-normes multiplicatives sur $A\left[T_{1}, \ldots, T_{n}\right]$, c'est-à-dire l'ensemble des applications

$$
|.|: A\left[T_{1}, \ldots, T_{n}\right] \rightarrow \mathbf{R}_{+}
$$

qui vérifient les propriétés suivantes :

(i) $|0|=0$ et $|1|=1$;

(ii) $\forall P, Q \in A\left[T_{1}, \ldots, T_{n}\right],|P+Q| \leq|P|+|Q|$;

(iii) $\forall P, Q \in A\left[T_{1}, \ldots, T_{n}\right],|P Q|=|P||Q|$.

Remarque B.1. Dans la définition proposée par Berkovich figure une condition supplémentaire qui fait intervenir une norme sur l'anneau $A$. Pour $a \in A$, posons

$$
\|a\|=\max _{\sigma \in \Sigma_{\infty}}\left(|\sigma(a)|_{\infty}\right),
$$

où $\Sigma_{\infty}$ désigne l'ensemble des plongements complexes du corps $K$ et $|.|_{\infty}$ la valeur absolue usuelle sur $\mathbf{C}$. La fonction $\|\|:. A \rightarrow \mathbf{R}_{+}$définit une norme sur $A$ et, lorsque l'on munit l'anneau $A$ de cette norme, la définition de Berkovich coïncide avec la nôtre. Signalons que, quelle que soit la norme dont on munit $A$ (sous réserve tout de même qu'elle soit sous-multiplicative et fasse de $A$ un espace complet), on obtient un espace contenu dans celui que nous avons défini.

Soit $x$ un point de $\mathbf{A}_{A}^{n, \text { an }}$. Il lui est associé une semi-norme multiplicative $|\cdot|_{x}$ sur $A\left[T_{1}, \ldots, T_{n}\right]$. L'ensemble $\mathfrak{p}_{x}$ des éléments sur lesquels elle s'annule est un idéal premier de $A\left[T_{1}, \ldots, T_{n}\right]$. Le quotient est un anneau intègre sur lequel la semi-norme $|.|_{x}$ induit une valeur absolue. Nous noterons $\mathscr{H}(x)$ le complété du corps des fractions de cet anneau pour cette valeur absolue. Nous noterons simplement $\mid$. | la valeur absolue sur le corps $\mathscr{H}(x)$, cela n'entraînant pas de confusion. La construction fournit un morphisme

$$
A\left[T_{1}, \ldots, T_{n}\right] \rightarrow \mathscr{H}(x) .
$$

L'image d'un élément $P$ de $A\left[T_{1}, \ldots, T_{n}\right]$ par ce morphisme sera notée $P(x)$. Avec ces notations, nous avons donc $|P(x)|=|P|_{x}$.

Munissons, à présent, l'espace analytique $\mathbf{A}_{A}^{n, \text { an }}$ d'une topologie : celle engendrée par les ensembles de la forme

$$
\left\{x \in \mathbf{A}_{A}^{n, \text { an }}|r<| P(x) \mid<s\right\},
$$

pour $P \in A\left[T_{1}, \ldots, T_{n}\right]$ et $r, s \in \mathbf{R}$.

Pour finir, nous définissons un faisceau d'anneaux 0 sur $\mathbf{A}_{A}^{n \text {,an }}$ de la façon suivante : pour tout ouvert $U$ de $\mathbf{A}_{A}^{n}$, an , l'anneau $\mathcal{O}(U)$ est constitué des applications

$$
f: U \rightarrow \bigsqcup_{x \in U} \mathscr{H}(x)
$$


qui vérifient les deux conditions suivantes :

(i) $\forall x \in U, f(x) \in \mathscr{H}(x)$;

(ii) $f$ est localement limite uniforme de fractions rationnelles sans pôles.

Dimension 0. Afin de rendre plus palpables les définitions précédentes, nous allons décrire explicitement $\mathbf{A}_{A}^{0, \text { an }}$, l'espace affine analytique de dimension 0 sur $A$, que nous noterons plus volontiers $M(A)$. Nous noterons $|.|_{\infty}$ la valeur absolue usuelle sur $\mathbf{C}$ et, pour tout idéal maximal $\mathfrak{m}$ de $A$, nous noterons $|\cdot|_{\mathfrak{m}}$ la valeur absolue m-adique normalisée. Du théorème d'Ostrowski, l'on déduit que les points de $M(A)$ sont exactement

(i) la valeur absolue triviale $|\cdot|_{0}$ (nous noterons $a_{0}$ le point associé);

(ii) la valeur absolue archimédienne $|\sigma(.)|_{\infty}^{\varepsilon}$ (nous noterons $a_{\sigma}^{\varepsilon}$ le point associé) pour tout toute classe de conjugaison de plongements complexes $\sigma$ de $K$ et tout élément $\varepsilon$ de $] 0,1]$;

(iii) la valeur absolue $\mathfrak{m}$-adique $|\cdot|_{\mathfrak{m}}^{\varepsilon}$ (nous noterons $a_{\mathfrak{m}}^{\varepsilon}$ le point associé) pour tout idéal maximal $\mathfrak{m}$ de $A$ et tout élément $\varepsilon$ de $] 0,+\infty[$;

(iv) la semi-norme $|\cdot|_{\mathfrak{m}}^{+\infty}$ (nous noterons $\tilde{a}_{\mathfrak{m}}$ le point associé) induite par la valeur absolue triviale sur le corps fini $A / \mathfrak{m}$ pour tout idéal maximal $\mathfrak{m}$ de $A$.

Nous pouvons également décrire la topologie de l'espace $M(A)$ ( $c f$. figure 2, tracée dans le cas où $K=\mathbf{Q}$ pour simplifier les notations, mais aisément généralisable). Pour cela, il suffit d'indiquer que chacune des branches tracée sur la figure est homéomorphe à un segment réel et qu'un voisinage du point central $a_{0}$ est une

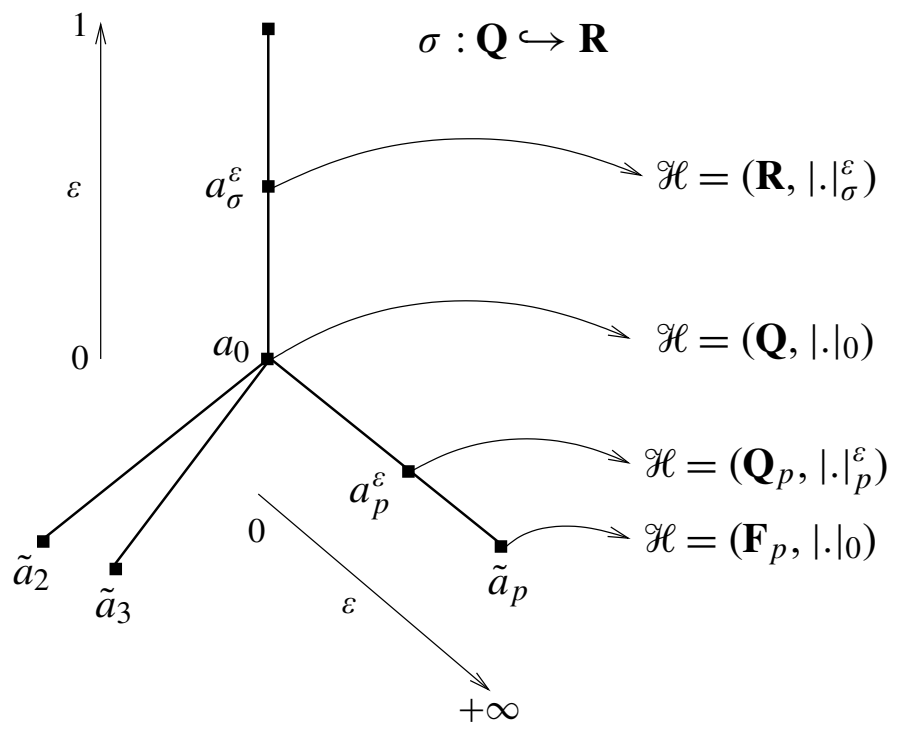

FigURE 2. L'espace $M(\mathbf{Z})$. 


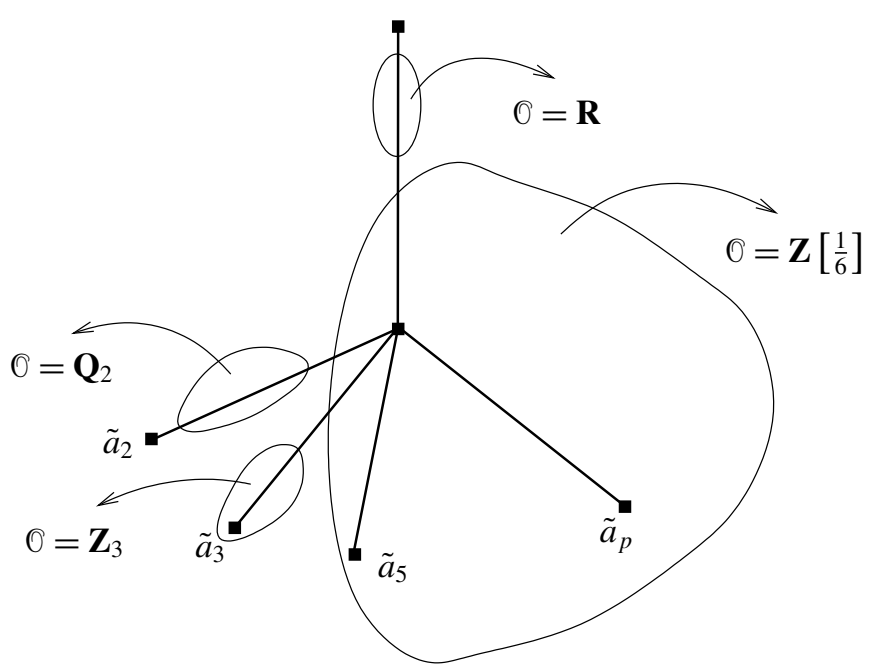

FigURE 3. Le faisceau structural sur $M(\mathbf{Z})$.

partie qui contient entièrement toutes les branches à l'exception d'un nombre fini, et qui contient un voisinage de $a_{0}$ dans chacune des branches restantes. Si l'on préfère, l'espace $M(A)$ possède la topologie du compactifié d'Alexandrov de la réunion disjointe de ses branches privées de $a_{0}$, le point $a_{0}$ jouant le rôle du point à l'infini.

Nous pouvons également décrire explicitement les sections du faisceau structural sur les ouverts de $M(A)$. Nous avons représenté les différents cas à la figure 3 , de nouveau dans le cas où $K=\mathbf{Q}$.

Dimension 1. Venons-en, à présent, à l'espace affine analytique de dimension 1 sur $A$. Nous noterons $T$ la coordonnée sur cet espace. Remarquons, tout d'abord, que le morphisme $A \rightarrow A[T]$ induit un morphisme de projection

$$
\pi: \mathbf{A}_{A}^{1, \text { an }} \rightarrow M(A) .
$$

Cela permet d'obtenir une description topologique de la droite de Berkovich sur $A$ : la fibre de $\pi$ au-dessus d'un point $x$ de $M(A)$ est isomorphe à la droite de Berkovich sur le corps $\mathscr{H}(x)$. Si $\mathscr{H}(x)=\mathbf{C}$, cette droite est isomorphe à l'espace $\mathbf{C}$ et, si $\mathscr{H}(x)=\mathbf{R}$, elle est isomorphe à son quotient par la conjugaison complexe. Nous ne chercherons pas à obtenir de description plus précise et nous contenterons d'indiquer quelques propriétés [Poineau 2008, théorèmes 4.4.1 et 4.5.5].

Théorème B.2. (i) L'espace $\mathbf{A}_{A}^{1 \text {,an }}$ est localement compact, métrisable et de dimension topologique 3.

(ii) L'espace $\mathbf{A}_{A}^{1, \text { an }}$ est localement connexe par arcs.

(iii) Le morphisme de projection $\pi: \mathbf{A}_{A}^{1, \text { an }} \rightarrow M(A)$ est ouvert. 
(iv) En tout point $x$ de $\mathbf{A}_{A}^{1, \text { an }}$, l'anneau local $O_{x}$ est hensélien, noethérien, régulier, de dimension inférieure à 2 et le corps résiduel $\kappa(x)$ est hensélien.

(v) Le faisceau structural 0 est cohérent.

Signalons encore que la droite de Berkovich sur $A$ satisfait au principe du prolongement analytique (ibid., théorèmes 4.4 .2 et 7.1.9, corollaire 4.4.5).

Théorème B.3. Soit $U$ une partie connexe de $\mathbf{A}_{A}^{1 \text {, an }}$.

(i) Le principe du prolongement analytique vaut sur U. En particulier, l'anneau $\mathrm{O}(U)$ est intègre.

(ii) L'anneau des sections méromorphes $M(U)$ est un corps.

(iii) Si $U$ est de Stein, le morphisme naturel Frac $(\mathcal{O}(U)) \rightarrow M(U)$ est un isomorphisme.

Rappelons ici ce que nous entendons par espace de Stein. Nous dirons qu'un espace localement annelé $\left(X, O_{X}\right)$ est de Stein s'il satisfait les conclusions des théorèmes $\mathrm{A}$ et $\mathrm{B}$ de $\mathrm{H}$. Cartan :

(A) pour tout faisceau de $0_{X}$-modules cohérent $\mathscr{F}_{F}$ et tout point $x$ de $X$, la fibre $\mathscr{F}_{x}$ est engendrée par l'ensemble des sections globales $\mathscr{F}(X)$;

(B) pour tout faisceau de $0_{X}$-modules cohérent $\mathscr{F}$ et tout entier $q \in \mathbf{N}^{*}$, nous avons $H^{q}(X, \mathscr{F})=0$.

Donnons quelques exemples de sous-espaces de la droite analytique $\mathbf{A}_{\mathbf{Z}}^{1 \text {,an }}$ qui sont des espaces de Stein (ibid., théorème 6.6.29).

Théorème B.4. Soient $V$ une partie ouverte et connexe de l'espace $M(A)$ et $s, t \in$ R. Soit $P(T)$ un polynôme unitaire à coefficients dans $O(V)$. Les parties suivantes de la droite analytique $\mathbf{A}_{A}^{1 \text {, an }}$ sont des espaces de Stein:

(i) $\left\{x \in \pi^{-1}(V)|s<| P(T)(x) \mid<t\right\}$;

(ii) $\left\{x \in \pi^{-1}(V)|| P(T)(x) \mid>s\right\}$.

Pour terminer, disons quelques mots des sections globales sur les parties de la droite analytique $\mathbf{A}_{A}^{1 \text {,an }}$. Sur les disques, elles s'expriment essentiellement en termes de séries dont les coefficients sont des fonctions sur $M(A)$. Considérons, par exemple, le disque ouvert relatif de rayon 1 :

$$
\mathbf{D}=\left\{x \in \mathbf{A}_{A}^{1, \text { an }}|| T(x) \mid<1\right\} .
$$

Le morphisme naturel $A[T] \rightarrow \mathcal{O}(\mathbf{D})$ induit un isomorphisme

$$
A_{1-} \llbracket T \rrbracket \stackrel{\sim}{\rightarrow} \mathrm{O}(\mathbf{D}),
$$


où $A_{1^{-}} \llbracket T \rrbracket$ désigne l'anneau constitué des séries de la forme

$$
\sum_{n \geq 0} a_{n} T^{n} \in A \llbracket T \rrbracket
$$

telles que le rayon de convergence de la série à coefficients complexes

$$
\sum_{n \geq 0} \sigma\left(a_{n}\right) T^{n}
$$

soit supérieur ou égal à 1 , pour tout plongement complexe $\sigma$ de $K$. On déduit cette description du théorème 3.2.16 de ibid.

À partir de la description des anneaux de sections sur les disques, nous pouvons déduire celle des anneaux locaux en certains points. Nous nous contenterons de deux exemples. Soit $\mathfrak{m}$ un idéal maximal de $A$. Notons $z_{\mathfrak{m}}$ le point 0 de la fibre de $\pi$ au-dessus du point $\tilde{a}_{\mathfrak{m}}$. D'après le corollaire 3.2.5 de ibid., le morphisme naturel $A[T] \rightarrow \mathrm{O}_{z_{p}}$ induit un isomorphisme

$$
\hat{A}_{\mathfrak{m}} \llbracket T \rrbracket \stackrel{\sim}{\rightarrow} \mathrm{O}_{z_{p}} .
$$

Notons $z_{0}$ le point 0 de la fibre de $\pi$ au-dessus du point $a_{0}$. D'après le corollaire 3.2.8 de ibid., le morphisme naturel $A[T] \rightarrow O_{z_{0}}$ induit un isomorphisme

$$
E \stackrel{\sim}{\rightarrow} \mathrm{O}_{z_{0}},
$$

où $E$ désigne l'anneau constitué des éléments $f$ de $K \llbracket T \rrbracket$ qui vérifient les propriétés suivantes :

(i) $\exists a \in A \backslash\{0\}, f(a T) \in A \llbracket T \rrbracket$;

(ii) pour tout plongement complexe $\sigma$ de $K$, le rayon de convergence complexe de la série $\sigma(f)$ est strictement positif;

(iii) pour tout idéal maximal $\mathfrak{m}$ de $A$, le rayon de convergence $\mathfrak{m}$-adique de la série $f$ est strictement positif (il suffit d'imposer cette condition pour les idéaux maximaux qui contiennent un élément $a$ possédant les propriétés décrites en (i).

\section{Remerciements}

La dernière partie de cet article a été rédigée au cours de l'année que j'ai passée à l'université de Ratisbonne. Je souhaite remercier Klaus Künnemann, qui m'a permis d'y séjourner, pour son accueil et ses encouragements. Ma gratitude va également à Antoine Chambert-Loir dont les conseils concernant la structure de cet texte m'ont permis, je l'espère, d'en accroître l'intérêt et la clarté. Merci également à Antoine Ducros de m'avoir communiqué ses notes sur les théorèmes GAGA. 


\section{Bibliographie}

[Berkovich 1990] V. G. Berkovich, Spectral theory and analytic geometry over non-Archimedean fields, Mathematical Surveys and Monographs 33, American Mathematical Society, Providence, RI, 1990. MR 91k :32038 Zbl 0715.14013

[Berkovich 1993] V. G. Berkovich, "Étale cohomology for non-Archimedean analytic spaces", Inst. Hautes Études Sci. Publ. Math. 78 (1993), 5-161 (1994). MR 95c :14017 Zbl 0804.32019

[Berkovich 1996] V. G. Berkovich, "Vanishing cycles for non-Archimedean analytic spaces", $J$. Amer. Math. Soc. 9 :4 (1996), 1187-1209. MR 97e :32037 Zbl 0988.14004

[Cartan 1951] H. Cartan, "Faisceaux analytiques sur les variétés de Stein : démonstration des théorèmes fondamentaux", dans Séminaire Henri Cartan 1951/52 (Exposé 19), vol. 4, 1951. Republié en 1955 par le département de mathématiques de MIT.

[Ducros 2009] A. Ducros, "Les espaces de Berkovich sont excellents", Ann. Inst. Fourier (Grenoble) $59: 4$ (2009), 1443-1552.

[EGA II 1961] A. Grothendieck, "Éléments de géométrie algébrique, II : Étude globale élémentaire de quelques classes de morphismes", Inst. Hautes Études Sci. Publ. Math. 8 (1961).

[Fried et Jarden 2008] M. D. Fried et M. Jarden, Field arithmetic, 3rd éd., Ergebnisse der Mathematik (3) 11, Springer, Berlin, 2008. MR 2009j :12007 Zbl 1145.12001

[Hakim 1972] M. Hakim, Topos annelés et schémas relatifs, Ergebnisse der Math. 64, Springer, Berlin, 1972. MR 51 \#500 Zbl 0246.14004

[Harbater 1984a] D. Harbater, "Convergent arithmetic power series", Amer. J. Math. $106: 4$ (1984), 801-846. MR 85j :13036 Zbl 0577.13017

[Harbater 1984b] D. Harbater, "Mock covers and Galois extensions", J. Algebra 91 :2 (1984), 281 293. MR 86c :13004 Zbl 0559.14021

[Harbater 1984c] D. Harbater, "Algebraic rings of arithmetic power series", J. Algebra 91 :2 (1984), 294-319. MR 86i :13007 Zbl 0578.13013

[Harbater 1987] D. Harbater, "Galois coverings of the arithmetic line", pp. 165-195 dans Number theory (New York, 1984-1985), édité par D. V. Chudnovsky et al., Lecture Notes in Math. 1240, Springer, Berlin, 1987. MR 88h :14020 Zbl 0627.12015

[Harbater 1988] D. Harbater, "Galois covers of an arithmetic surface", Amer. J. Math. 110 :5 (1988), 849-885. MR 90e :14013 Zbl 0683.14004

[Harbater 2003] D. Harbater, "Patching and Galois theory", pp. 313-424 dans Galois groups and fundamental groups, édité par L. Schneps, Math. Sci. Res. Inst. Publ. 41, Cambridge Univ. Press, 2003. MR 2004j:14030 Zbl 1071.14029

[Kiehl 1967] R. Kiehl, "Der Endlichkeitssatz für eigentliche Abbildungen in der nichtarchimedischen Funktionentheorie”, Invent. Math. 2 (1967), 191-214. MR 35 \#1833 Zbl 0202.20101

[Köpf 1974] U. Köpf, "Über eigentliche Familien algebraischer Varietäten über affinoiden Räumen”, Schr. Math. Inst. Univ. Münster (2) Heft 7 (1974), iv+72. MR 54 \#10657 Zbl 0275.14006

[Liu 1995] Q. Liu, “Tout groupe fini est un groupe de Galois sur $\mathbf{Q}_{p}(T)$, d'après Harbater", pp. 261-265 dans Recent developments in the inverse Galois problem (Seattle, 1993), édité par M. D. Fried et al., Contemp. Math. 186, Amer. Math. Soc., Providence, RI, 1995. MR 96h :12006 Zbl 0834.12004

[Moret-Bailly 2001] L. Moret-Bailly, "Construction de revêtements de courbes pointées", J. Algebra 240 :2 (2001), 505-534. MR 2003a:14042 Zbl 1047.14013 
[Poineau 2008] J. Poineau, "La droite de Berkovich sur Z”, preprint, 2008. À paraître dans Astérisque (2010). arXiv 0809.2880

[Pop 1996] F. Pop, "Embedding problems over large fields", Ann. of Math. (2) 144 :1 (1996), 1-34. MR 97h :12013 Zbl 0862.12003

[Saltman 1982] D. J. Saltman, "Generic Galois extensions and problems in field theory", Adv. in Math. 43 :3 (1982), 250-283. MR 84a :13007 Zbl 0484.12004

[Serre 1955-1956] J.-P. Serre, "Géométrie algébrique et géométrie analytique", Ann. Inst. Fourier, Grenoble 6 (1955-1956), 1-42. MR 18,511a Zbl 0075.30401

[SGA1 1971] A. Grothendieck (éditeur), Revêtements étales et groupe fondamental, Lecture Notes in Mathematics 224, Springer, Berlin, 1971. MR 50 \#7129

Communicated by Jean-Louis Colliot-Thélène

Received 2009-05-29 Revised 2009-08-31 Accepted 2009-09-30

jerome.poineau@math.unistra.fr Institut de recherche mathématique avancée, 7, rue René Descartes, 67084 Strasbourg, France http://www-irma.u-strasbg.fr/ poineau/ 